\title{
AUTHOR INDEX OF 36TH WORLD CONGRESS OF ENDOUROLOGY by abstract number
}

\section{A}

ABARZUA-CABEZAS F, UP5-54

ABAZA R, MP16-5, MP16-18, MP25-16, UP2-50, UP5-1

ABBOTT JE, MP12-20, MP26-2, MP32-17, VS1-6

ABD ELZAHER M, MP11-15

ABDALLAH MM, UP3-5

ABDALLH MM, MP11-4

ABDEEN TM, MP9-1

ABDEL GHANY M, MP21-1

ABDEL WAHAB M, MP21-1

ABDELBARY N, UP4-9

ABDELBAST M, MP9-14, UP3-60

ABDELFADIL S, MP31-16

ABDEL-GAWAD M, MP2-6, MP31-11

ABDEL-KARIM A, UP2-10, VS14-5, VS14-9

ABDELHADY M, MP28-9

ABDELHALIM A, MP21-14

ABDELHAMID A, MP12-12, MP24-7, MP27-6

ABDELHAMID U, MP28-5

ABDELKAREEM M, UP5-5

ABDELMALEK B, MP9-8

ABDELMOTELEB H, MP1-15

ABDELSALAM A, UP1-9

ABDUL-MUHSIN H, UP3-11, UP5-27, VS13-4

ABDULKAREEM AS, UP4-35

ABDULLATEEF M, MP9-19, UP4-36

ABDULLAYEV E, UP3-18

ABE T, MP17-16, MP19-2, MP19-6, MP19-11, UP1-57, UP3-36, UP4-59, UP5-28

ABEERY HM, UP5-60

ABO EL-ELA A, MP30-4

ABOELFOTOH A, VS14-9

ABOL-ENEIN H, MP9-14

ABOU ZAMEL A, MP21-1

ABOU-TALEB A, MP11-15

ABOUL ELA W, MP21-1

ABOUMARZOUK O, MP4-18, MP9-3, MP13-17, MP31-9, UP5-11, UP5-48, UP6-43

ABOURBIH S, MP7-3, MP7-4, MP15-1, UP4-1

ABOURMARZOUK O, UP5-50

ABRAHAM JA, MP17-15

ABRAHAO R, MP16-11

ABU ARAFEH W, MP9-1

ABU HAMAD R, UP1-31

ABU-GHANEM Y, MP10-22, UP5-31, UP6-59

ABULKHAIR A, VS14-5, VS14-9

ABUSHAMMA F, MP13-17, UP5-48

ACAR C, MP33-8

ACHINAS S, MP5-3

ACQUATI P, MP11-22, MP27-17, UP3-16, UP3-28, UP3-58

ADAMOU C, MP3-9, MP21-2, UP6-30
ADAMS F, MP22-9

ADASONLA K, UP5-40

ADDAR A, MP13-19

ADELOYE DO, MP3-2, UP1-2

ADIKI A, MP22-7

AGARWAL DK, MP2-1, MP31-1, MP33-19, UP1-14,

UP3-19, VS15-2

AGARWAL P, VS18-1

AGGARWAL A, MP14-22

AGRAWAL M, MP7-1

AGRAWAL S, UP6-35

AGUDELO J, MP16-6, MP16-15, MP23-18, MP30-10, UP2-12

AHLERING TE, MP15-12, MP22-19, UP5-19, UP5-20

AHLUWALIA P, MP20-12, MP30-17, VS7-9, VS17-6

AHMAD NA, UP4-4

AHMAD T, MP28-3

AHMED A, MP29-21

AHMED K, MP19-1, MP19-2, MP19-6, MP19-15, MP19-18, MP21-3, UP3-36, UP4-58, UP5-59

AHMED M, MP13-18, MP18-6, MP22-6, MP23-3, MP25-20, UP2-7, UP5-30, VS6-8, VS6-13, VS7-5, VS18-6

AHMED S, MP11-15

AHOUAGI A, MP16-11

AIMAR R, MP15-4, UP2-33, UP5-38

AJAYI L, MP7-16, MP7-17, MP28-18, UP4-2, UP4-19, UP4-20

AK E, VS10-7

AKATSUKA J, UP2-30

AKAY A, MP9-20

AKBAL C, UP4-31, VS6-9

AKCA O, MP20-7, VS9-6

AKDEMIR O, MP17-7

AKDEMIR S, MP24-4

AKDENIZ F, VS6-2, VS13-7

AKERS A, MP33-9

AKHA WEAS I, UP1-4

AKHTER S, MP12-8, MP24-16, MP31-20, UP6-27

AKHVLEDIANI N, MP11-21

AKINBOLA IA, UP1-2

AKINCI A, MP12-2

AKOPYAN G, VS1-3

AKPINAR H, MP16-4, VS8-1, VS10-1

AKSENOV LI, MP28-1

AKSOY F, VS5-1

AL ANSARI A, UP5-5

AL AOWN A, MP3-9

AL JANABI H, MP19-1, MP21-3, UP4-58

AL K, UP1-16

ALTINAWI B, MP8-13

ALAEV Y, VS17-10

ALAHMAD A, MP26-9

ALAJRAWI F, MP29-21 
ALALI K, MP26-9

ALAM A, MP12-4, MP17-19

ALAM M, MP24-16, MP31-20, UP6-27

ALAMI M, VS2-7, VS8-11

ALAMIRI A, MP29-21

ALANBUKI A, MP23-9

ALANZI T, MP26-9

ALBANESI L, MP6-4, MP23-20, MP30-12

ALBAYRAK A, MP32-9, UP3-18

ALBERSHEIM-CARTER J, MP10-3

ALBO G, MP22-1, UP4-8, VS15-3

ALCARAZ A, MP6-3, MP20-17, UP2-56

ALDARRAB R, MP13-19

ALDOUKHI AH, MP24-6, MP24-11, MP27-1, UP4-32, VS1-5, VS2-6

ALEJANDRO G, UP6-49, VS11-7, VS11-9

ALEKSANDROV I, VS13-9, VS14-4

ALENIZI AM, VS10-2

ALEXANDRESCU E, UP5-51

ALGABA F, MP21-11

ALGER J, MP4-17

AL-HASHIMI A, MP19-7, UP3-37

AL-JABIR A, MP19-1, MP19-2, MP21-3, UP4-58

AL-KRUSH A, UP1-4

AL-OMAR O, UP3-40, VS6-3

AL-RUWAISHED M, MP28-5

AL-SHAIJI T, MP28-5

AL-TERKI A, MP28-5, MP29-21

ALHARBI M, MP3-3

AL-HADI A, MP31-19

ALHUNAIDI O, MP29-21

ALI A, MP12-12, MP13-11, MP24-7, MP27-6

ALI S, VS1-3

ALI Z, MP13-1, MP32-19

ALI-EL-DEIN B, MP2-6

ALINEJAD M, UP4-23

ALKAZEMI MH, MP9-11

ALKHARJI A, VS10-2

ALKHUDAIR WK, MP25-14

ALLAHVERDIYEV E, UP4-26, UP5-49

ALLARD R, MP33-9

ALLEN C, MP7-15

ALLEN D, MP7-16, MP7-17, MP28-18, UP4-2, UP4-19, UP4-20

ALLEN J, MP13-20

ALLEN R, VS13-5

ALLEN S, MP14-7, MP14-15

ALLEVA G, MP15-4, UP2-33

ALLONA A, VS11-5

ALMANSOUR M, VS10-2

ALMUKHTAR S, UP1-4

ALMÚSTER DOMÍNGUEZ S, VS2-2

ALMUSTER DOMÍNGUEZ S, VS15-9

ALOISI P, MP6-4, MP23-20, MP30-12

ALOM M, MP33-21

ALOMAR MA, UP5-60

ALONI S, UP1-20

ALOOSH M, UP3-39

ALOTAIBI MF, MP25-14

ALOTHMAN KI, MP25-14

ALQATTAN Y, MP28-5

ALRUMAIHI K, UP5-5

ALSAYEGH N, MP2-6
ALSHALALFA M, MP1-1

ALSHARA L, MP29-2

ALSHOHAYEB I, MP13-19

ALSYOUF M, MP7-3, MP7-4, MP15-1, UP4-1

ALTER J, MP4-1

ALTHAUS A, MP7-2

ÁLVAREZ-OSSORIO J, VS11-5

ALY S, VS18-6

ALZAHRANI HM, MP25-14

AMANO N, MP22-18

AMARO J, MP22-3, MP25-22

AMASYALI A, MP7-3, MP7-4, MP15-1, UP2-31

AMELI M, MP26-22

AMER T, MP31-9

AMINOLTEJARI K, MP9-16

AMIR M, UP1-28

AMIRHASANI S, UP4-7

AMLANI J, UP3-27, UP6-50

AMMANI A, VS2-7, VS8-11

AMPARORE D, MP6-1, MP14-10, MP15-4,

UP2-33, UP5-38

ANACLETO S, MP8-4, UP4-21

ANANDAKUMAR A, UP3-56

ANASTOS H, MP4-3, MP23-13, UP1-35

ANBARASAN R, MP9-2

ANCESCHI U, MP30-7

ANDER H, MP4-4, MP4-5, MP24-9, UP1-36

ANDERSON B, MP3-18, MP8-7, MP16-12, MP25-7,

UP6-41, VS1-11

ANDERSON C, UP3-30

ANDERSSON S, MP29-18

ANDONIAN S, MP24-15, UP3-39, UP4-60

ANDREASSEN KH, MP2-10, MP2-11

ANDRÉN O, MP29-18

ANDREWS HO, MP17-18, MP17-21

ANDRIOLE G, MP4-7, MP4-8, MP18-11

ANDRONOV A, MP12-3, MP23-19

ANELE U, MP16-13

ANGERRI O, VS1-9

ANGURALA N, MP4-6

ANKEM MK, MP11-10, UP5-39

ANNIS J, UP1-28

ANSON K, UP1-49

ANTMEN M, MP1-8

ANTOINE S, MP28-2

ANTONELLI J, MP8-17, MP8-18, MP27-13, MP28-3,

MP28-20, UP6-22

ANTON-CULVER H, UP3-50

ANTONIO A, VS11-7, VS11-9

ANTONUCCI M, MP1-5

ANTUNES H, MP28-4

ANWAR A, MP12-12, MP24-7, MP27-6

AOKI H, MP9-18

AOUN R, MP12-14

APAKAMA I, MP4-6

ARAB H, MP21-14

ARAKI A, MP16-1, MP19-10, MP19-12

ARAKI M, MP9-18, MP15-16, MP25-9, MP25-11, MP30-16,

UP2-22, UP2-39, UP6-55

ARAUJO L, MP22-3

ARCALA R, MP29-3

ARCE S, MP2-3, MP2-4, UP1-15

ARDA E, UP4-37, UP6-12 
ARENA G, MP32-2, MP32-10

ARGHITTU M, MP22-1

ARGUN B, MP6-11, MP18-1, MP20-10, UP1-48, VS9-7, VS10-1

ARGUN O, MP16-2, VS7-7

ARMANYOUS S, MP4-11, MP16-15, MP23-18, MP30-10, UP2-1, UP2-12, UP5-2

ARMAZ VILANEDA M, UP1-53

ARMAZ VILLANEDA M, MP10-18

ARMITAGE J, MP7-2, MP7-12, MP7-22, MP32-15

ARON M, VS7-1

ARSLAN I, MP24-2

ARSLAN M, MP24-4, MP32-13

ARUMUHAM V, MP7-15, MP14-7, MP14-15

ARZATE-SORIANO R, MP2-8

ASADPOUR A, UP4-10

ASAI S, UP2-43

ASAKURA T, UP2-38

ASALEYE CM, MP3-2, UP1-2

ASFUROGLU A, MP29-20

ASHIZAWA T, MP28-11

ASHMAWY A, MP30-4

ASHOU R, MP12-14

ASLZARE M, UP4-10

ASLAN Y, MP17-7, MP23-7, MP24-4, MP29-20

ASPLIN J, MP10-9, MP10-20, MP28-2, MP31-8

ASSAF E, MP12-14

ASSEM A, MP30-4

ASSIMOS DG, MP2-20, MP2-21, MP10-21, UP6-40, UP6-57

ASUTAY M, VS6-9

ATALLAH W, MP24-22, MP29-5, MP32-5, UP6-6, UP6-20

ATEF L, MP9-8

ATHANASIADIS G, MP19-9

ATICI A, VS1-7

ATIS G, MP25-8

ATMOKO W, MP12-7, MP26-1

ATUG F, MP15-2, UP2-32

ATWA A, MP9-14

ATWELL TD, MP18-9

AUER AO, MP7-5

AUGUSTINE J, MP4-11

AUTORINO R, MP16-13

AVANT RA, MP33-21, UP3-33, UP3-34, VS12-6, VS16-7, VS16-8

AVDA Y, MP8-1, MP8-15, UP6-13, UP6-37

AVERCH T, MP8-5, MP22-10, MP22-15, MP28-3, MP28-20, UP6-11

AVILA HERRERA P, MP24-3

AYAD ML, MP5-16

AYBAL H, MP3-8, MP33-16, VS3-7, VS3-8, VS10-7, VS13-8, VS13-10

AYDIN A, MP19-1, MP19-2, MP19-6, MP19-15, MP19-18, MP21-3, UP3-36, UP4-3, UP4-58, UP5-59

AYDIN B, MP4-4, MP4-5, UP1-36

AYDOGAN T, MP3-8, MP33-16, VS13-10

AYKANAT C, MP17-7, MP24-4, MP29-20

AYKANLI E, UP3-18

AYSEGUL E, MP33-17

AYTAÇ, UP2-32

AYTAÇ O, MP15-2

AYYASH O, MP8-5

AZAD M, MP26-16, MP26-17

AZHAR RA, MP25-14

AZLI M, UP4-38
B

BAARD J, MP5-17, MP21-16, MP24-17

BABALOLA RN, UP1-2

BABAYIĞIT M, MP12-2, MP33-18

BACH T, UP3-35

BACO E, MP16-3

BADANI K, MP16-5, MP16-18, MP18-3, MP25-16, UP2-50, UP5-1, UP5-6, VS10-6, VS18-9

BADAWY H, MP21-1

BADAWY M, MP9-14, UP1-9, UP3-60

BADMUS TA, MP3-2, UP1-2

BAE W, MP12-11, MP26-6

BAGCIOGLU M, MP26-10

BAGLEY DH, MP27-2, VS5-11, VS6-5

BAHADIR S, UP1-22

BAHILO MATEU P, VS15-14

BAHÍLO MATEU P, VS2-8

BAHLER CD, MP4-1, MP4-2, VS10-9

BAILEY M, MP5-1, MP29-11

BAILIE J, MP13-21

BAKAVICIUS A, MP23-14

BAKDIK S, VS5-1

BALAI E, MP31-10

BALAJI G, UP4-5

BALAJI S, MP7-6, MP18-2, MP31-22, UP6-52, VS12-1

BALAKRISHNAN A, UP4-5

BALAN G, MP2-7, MP3-4, MP24-1, UP1-5, UP4-33, UP4-45, UP5-51

BALCI M, MP17-7, MP24-4, MP29-20

BALDWIN DD, MP7-3, MP7-4, MP15-1, UP2-31, UP4-1

BALEN A, MP8-9, MP32-6

BALTACI S, MP12-2

BAMBERGER J, MP29-5, MP32-5

BANAPOUR P, MP15-3, UP5-56

BANAS M, VS5-9

BANDARI J, MP33-6

BANDINI M, MP30-6

BANG S, VS18-3

BANG W, MP3-16, UP4-13

BARABAS M, MP7-5

BARBER N, MP6-1

BARCLAY T, MP29-9

BAREH A, MP4-18

BARGHOUTHY Y, MP29-10, MP32-18

BARLING J, MP33-9

BARNETT H, MP27-7

BARNETT P, MP3-12

BARR A, MP9-3

BARRASS B, MP17-19

BARRET E, MP20-13, MP23-14

BARROS RODRÍGUEZ J, VS2-2

BAŞATAÇ C, MP16-4, VS8-1, VS10-1

BASILE G, MP20-17, MP21-11, MP21-12, UP2-56, VS15-8

BASIRATNIA M, MP9-4

BASIRI A, MP32-1, VS4-4

BASRALIAN K, MP20-15, UP3-25

BASSI P, MP1-4, UP1-8

BASTIANPILLAI C, MP31-10

BASTON E, MP9-21

BASULTO-MARTÍNEZ MJ, UP6-14

BATAGELLO C, MP26-20, MP29-2

BATAI K, MP1-10, MP1-11, MP3-6, UP2-51

BATTER T, MP26-20

BAUER N, UP1-49 
BAUERNFREUND Y, UP3-55

BAUMERT H, MP11-14, UP3-59

BAUZA QUETGLAS J, VS8-10

BAY D, MP16-3

BAYAR G, MP13-6

BAYNE D, MP7-7, MP10-18, MP33-2, MP33-3

BAZIN D, UP1-24

BEARRICK E, MP10-3

BECHIS SK, MP8-8, MP29-2

BECKER B, MP2-13, MP3-1, MP3-5, MP3-17, MP6-17, MP10-7, MP11-5, MP11-9, UP3-13, UP6-46

BEDÜK Y, MP12-2

BEHKAM B, MP22-10

BEIKO D, MP33-9

BEJAN C, MP31-6

BEKSAC A, MP1-1, MP16-5, MP18-3, MP18-16, MP18-19, MP25-16, UP5-1, UP5-6, UP5-8, VS10-6, VS18-9

BELAL M, MP14-4, MP14-14

BELANI J, MP29-3

BELEW D, MP16-22

BELL J, MP24-14

BELL R, MP1-11, MP3-6

BELLINZONI P, UP4-24, VS1-12

BELTRAMI P, MP5-2, MP21-4

BELTRAN-SUAREZ E, VS2-3

BELVIRANLI M, VS5-1

BENABDALLAH J, MP25-7

BENDIGERE T, VS8-5

BENFANTE N, MP30-19

BEN-YA'ACOV D, UP4-51

BENRABAH R, UP4-38

BENTANI N, VS2-7

BERARDINELLI F, MP16-13, MP23-6

BERDEMPES M, UP6-4

BERG R, MP16-3

BERG W, VS16-9

BERGER A, MP31-2

BERGER I, MP13-5, UP3-48

BERGERSEN A, MP1-10

BERGMAN J, MP33-4

BERI A, MP29-10, MP32-18

BERMAN I, MP13-21

BERNARDI D, MP32-2, MP32-10

BERNSTEIN MA, VS15-4

BERQUIST S, UP5-4

BERRY CJ, VS14-1

BERTA G, UP3-12, VS3-13, VS14-7

BERTHE LL, MP5-4, MP5-11, MP5-20, MP24-5, VS1-4 BERTINI R, MP16-17

BERTO C, MP21-4

BERTOLO R, MP16-6, MP16-13, MP16-15, MP18-4, MP23-18, MP25-12, MP25-13, MP30-10, UP2-1, UP2-12, UP2-45, UP5-2, VS5-10, VS8-10

BESARANI D, UP3-32

BESHENSKY D, MP2-15

BEST SL, MP10-11

BETTIN L, MP5-2, MP21-4

BEZRUKOV E, VS7-10, VS12-4, VS15-10

BHALODI AA, MP24-14

BHANDARI A, MP16-5, MP25-16, UP2-50, UP5-1

BHAT N, MP4-8

BHIRUD P, VS3-9, VS5-8

BIALOWONS W, UP3-45

BIDNUR S, UP1-29
BIFFAR D, UP2-51

BIGLEY J, MP22-15

BIHORAC A, MP12-19

BILEN C, MP33-8

BILIGERE S, MP27-21, UP6-18

BILLIET I, MP23-7

BINDAYI A, MP18-21, MP23-6

BIRD E, MP22-21, MP33-15

BIRD N, MP2-3

BIRD V, MP2-2, MP2-3, MP2-4, UP1-15, UP5-41, UP6-33

BIRD VG, MP10-16, MP12-19, MP28-3, MP28-20

BIROWO P, MP12-7, MP26-1

BISHOP C, UP3-6

BIYANI C, MP19-9

BIYANI S, MP13-13, MP13-14

BJAZEVIC J, MP10-1, UP1-16

BJURLIN M, MP17-12, VS16-5

BLAIBEL M, MP15-1, UP2-31

BLANKSTEIN U, MP19-7, UP3-37

BLEZIEN O, MP11-22, MP27-17, UP3-16, UP3-28, UP3-58

BLOCH A, MP23-1, UP5-4

BLOOM J, MP11-16, MP12-6, VS18-1

BLUEBOND-LANGNER R, MP14-9

BLUM K, UP2-50

BOAZ RJ, VS14-1

BODEN A, MP32-20

BOERI L, MP5-7, MP22-1, MP31-3, UP1-44, UP1-45,

UP4-8, VS15-3

BOLGERI M, UP1-49

BOLLITO E, UP5-38

BOLOMYTIS S, MP7-8, MP13-13, MP13-14, UP1-17

BOLTON D, MP29-16

BON D, MP27-4

BONDAD J, MP13-7

BONI A, UP5-14

BORA GS, UP1-3, VS2-1

BORDIER B, UP3-23

BÖRGERMANN C, UP3-45

BORGLUM N, MP8-6, UP6-19

BORHANI A, MP22-15

BORIN J, MP17-12

BORISENKO G, MP30-20

BOROFSKY MS, MP4-21, MP10-3, MP21-19

BORONAT TORMO F, VS2-8, VS15-14

BOROWIK M, VS6-14

BOS D, MP20-14

BOSE P, UP5-55

BOSTANCI Y, UP5-46, VS6-2, VS8-4, VS13-1,

VS13-7, VS14-11

BOTEJUE M, MP14-5, VS16-10

BOURGI A, MP11-14, UP3-59

BOWEN DK, MP4-16, MP9-5

BOWER P, MP8-9, MP32-6

BOYD C, UP6-40, UP6-57

BOZORGMEHRI S, MP4-14, MP10-16, MP12-19

BRACAMONTE E, MP1-11, MP3-6, MP4-9

BRACCHITTA D, MP30-9

BRADLEY CR, MP7-9, MP12-13, MP26-4

BRAMBILLA R, UP1-44, UP1-45

BRAUN A, MP31-4, UP6-8

BRAVO G, MP2-8

BRAVO-BALADO A, MP3-7, UP1-6

BREDA A, MP20-17, MP21-11, MP21-12,

UP2-56, VS15-8 
BREHMER M, MP24-17, UP5-12

BRES-NIEWADA E, MP19-4, UP1-22

BREUER G, UP3-45

BREWIN J, MP29-4, UP1-28

BRINKMANN R, MP5-15

BROCHERT A, UP1-39

BROWN P, UP6-45

BRUNCKHORST O, UP5-59

BRUNIN D, VS12-4

BRYCKAERT P, MP14-1, MP30-9

BRYSON CF, MP13-12, UP3-20

BUCKLEY J, VS12-5

BUDAYA TN, MP21-20

BUDIA ALBA A, VS2-8, VS15-14

BUDIA A, MP27-12

BULAI C, MP2-7, MP3-4, MP24-1, MP29-1, UP1-5, UP4-39, UP5-51

BULTITUDE M, MP19-15, UP5-42, UP5-59

BURGESS N, MP7-2, MP7-12, MP7-22, MP32-15

BURTON J, UP1-16

BUTTICE S, MP19-4, MP24-9, UP1-22

BYLUND JR, MP24-14, MP25-2, MP28-7, MP28-8, UP3-51

BYUN K, MP15-5

BYUN S, MP16-21, MP30-22, UP3-38

C

CABANAS C, MP23-15, UP1-59

CABRAL J, UP5-53

CADEDDU JA, MP19-8, MP23-11, UP1-34, UP1-38, VS5-6

CADILLO-CHAVEZ RG, MP15-6, MP16-7, MP33-10, UP2-4, VS7-11, VS18-7

CAI H, MP1-21, UP2-42

CAICEDO J, MP3-7, UP1-6

CAKIROGLU B, UP4-37, UP6-12

CAKMAK S, MP13-6

CALIM A, UP3-18

CALIO BP, MP27-2, VS5-11, VS6-5

CALLAS P, MP29-9

CALVO VAZQUEZ I, UP4-11, VS6-11, VS18-8

CAMPOLEONI M, UP1-44, UP1-45

CAMPOS SALCEDO J, MP21-8

CAMPOS JR, UP1-1

CAMPOS-NEGRETE A, MP2-8

CAMPWALA Z, MP15-1, UP2-31

CANALES BK, MP10-16

CANNELLA R, MP22-15

CANTELLANO OROZCO M, UP4-11, VS6-11, VS18-8

CANVASSER N, MP8-17

CAO M, MP22-2

CAPITANIO U, MP16-17, MP18-21

CAPOCCIA E, MP21-17, MP22-16, VS18-11

CAPRETZ T, MP2-14

CARBALLIDO RODRIGUEZ J, VS2-5

CARBALLIDO J, VS16-14

CARBALLIDO-RODRÍGUEZ J, MP25-3, UP5-3, VS17-5

CARBALLO QUINTÁ M, VS2-2, VS15-9

CARDENAS-ORTIZ A, MP22-3, MP25-22

CARINI M, MP16-13

CARLOS E, MP10-20, MP27-18, MP29-12, UP1-55, UP6-1, VS10-8

CARMIGNANI L, MP11-22, MP27-17, MP30-6, UP3-16, UP3-28, UP3-58

CARNEIRO A, UP1-1, VS8-3

CARNES A, MP16-8, MP16-9
CARRICK M, MP4-3, MP23-13

CARRIER S, MP3-3, MP24-15, MP31-19

CARRIERE SP, VS16-3

CARRION R, VS3-12

CARTER A, VS15-15

CARUGO D, UP1-26

CASALE AS, UP5-43

CASKURLU T, MP25-8

CASTANHEIRA DE OLIVEIRA M, VS8-6

CASTILLO JC, MP15-17

CASTILLÓN VELA IT, MP16-20, MP25-3, MP25-4, MP33-10, UP5-3, VS16-14, VS17-5

CASTLE E, VS13-4

CASTLE J, MP25-17

CASTRO IGLESIAS, VS15-9

CATARI MALLEA A, UP5-54

CATARINICCHIA S, MP27-8

CATHCART P, MP22-5

CATHELINEAU X, MP23-14

CATTANEO F, MP5-2

CATTANEO G, MP14-10, UP5-38

CATTERWELL R, MP25-18

CAVALANTI A, MP13-9

ÇELEN S, MP32-13

CELENTANO G, MP7-15, MP14-7

CELIA A, UP2-3

CELIK H, VS13-1

ÇELIK O, UP6-9

CEPEDA M, MP27-12

CESARETTI J, UP1-39

CETTI R, MP7-5

CHA JD, UP1-1

CHADERTON M, MP2-2

CHALHOUB K, MP12-14

CHALLACOMBE B, MP11-18, MP17-2, MP22-5, MP25-10, MP25-18

CHAN E, MP30-14

CHAN J, MP9-16

CHANDNA A, VS8-7

CHANDRA A, UP5-42

CHANG C, UP6-51, VS12-2

CHANG H, VS11-11

CHANG HC, MP29-11, UP1-43

CHANG J, UP3-50

CHANG K, MP16-13

CHANG S, MP11-2, MP31-2

CHANG T, UP6-54

CHANG Y, MP28-22, UP5-18

CHAPARALA H, MP10-5

CHARALAMPOGIANNIS N, MP5-3

CHATZIGIANNIS C, UP3-45

CHAU Q, MP17-13, VS13-2

CHECCUCCI E, MP14-10, MP15-4, UP2-33, UP5-38

CHEE J, UP3-6

CHELGHAF I, MP12-21

CHEN C, UP2-25, VS9-3

CHEN D, MP12-10, MP26-8

CHEN F, UP6-7

CHEN FK, UP1-1

CHEN H, MP22-2

CHEN K, MP25-21, VS4-5

CHEN KW, UP3-2

CHEN M, MP4-22, MP17-4, UP2-11, UP4-14, UP6-25, VS14-12 
CHEN S, MP1-21, MP4-20, MP4-22, MP17-4, MP21-9, MP21-10, MP26-21, MP33-22, UP1-11, UP2-11, UP2-42, UP4-27

CHEN W, VS2-4

CHEN X, UP5-41, UP6-7

CHEN Y, MP3-10, MP14-13, MP18-18, UP1-11, UP2-25, UP4-6, UP4-40, UP4-41, UP5-18, UP6-31

CHENEY S, UP3-11

CHENG C, UP5-7

CHENG G, MP1-12

CHENG J, MP7-4, MP15-1, UP3-6, UP4-1

CHENG L, MP4-2, VS7-13

CHENG W, UP6-15, UP6-54

CHENG Y, UP5-24

CHEON S, UP1-42

CHERNYSHEVA D, UP3-41

CHERTIN B, MP14-21, UP2-5

CHETWOOD A, UP6-35

CHEUNG S, UP5-58, UP6-38

CHEVINSKY M, MP8-2, MP8-3, UP1-23

CHEW B, MP28-22

CHEW BH, MP2-17, MP5-19, MP8-19, MP9-16, MP19-2, MP28-3, MP28-20, UP1-29

CHI T, MP7-7, MP10-18, MP28-3, MP28-20, MP33-2, MP33-3, UP1-53

CHIANG C, MP16-19

CHIANG G, MP14-19, VS6-1

CHIANG P, MP18-17

CHIAPELLO G, MP32-2, MP32-10

CHIBA K, MP15-8

CHIBA S, MP25-5, UP2-46, VS8-12

CHINA T, MP28-11

CHIRON PH, MP5-4, MP5-6, MP5-11, MP5-14, MP5-20, MP24-5, VS1-1, VS1-4

CHIRUVELLA M, VS8-5

CHITHIRAMOHAN A, MP14-4, MP14-14

CHIU A, MP17-20, VS11-10

CHIU H, VS12-2

CHIU P, MP30-14

CHIU Y, UP6-15, UP6-54

CHO B, VS5-3

CHO EY, VS16-6, VS16-13

CHO H, MP12-11, MP17-17, MP26-6

CHO I, MP15-13, MP18-13, MP20-11, MP25-19, UP5-10

CHO K, MP31-15, MP32-12

CHO M, MP13-15, MP29-15

CHO S, MP3-16, MP13-15, MP17-17, MP29-15, UP4-13

CHO W, MP31-13

CHOE H, MP10-4, MP26-3

CHOI C, UP5-21

CHOI H, MP18-12, UP5-21

CHOI J, MP10-4, MP21-18, UP3-50, UP4-42

CHOI S, MP12-11, MP15-5, MP17-17, MP21-6, MP25-6, MP26-6, MP32-4, UP3-53

CHOI T, MP22-20

CHOI W, UP3-47

CHOI Y, MP12-11, MP15-13, MP17-17, MP18-13, MP20-11, MP25-19, MP31-15, MP32-12, UP5-9, UP5-10, UP5-22, VS7-12

CHONDROS K, MP30-9

CHONG J, UP6-20

CHONG Y, UP5-43

CHOO M, MP3-16

CHOO S, MP21-18, UP6-44
CHOONG S, MP7-15, MP14-7

CHOPRA S, VS7-1

CHOTIKAWANICH E, MP8-14, VS1-8

CHOU S, UP1-18, VS12-9

CHOUDHURY M, MP11-16, MP12-6

CHOUSIANITIS Z, UP4-44

CHOW AK, MP18-5

CHOW K, MP9-21

CHOW M, MP16-10

CHOW P, VS11-11

CHRISTIANSON S, MP31-4, UP6-8

CHRISTMAN M, MP17-5

CHU C, MP9-15

CHU K, UP3-6

CHUANG T, UP6-15

CHUEH S, VS17-2

CHUI K, UP4-16

CHUN S, MP15-5, MP32-4

CHUNG B, MP14-3, MP18-14, MP31-2, UP6-16

CHUNG E, UP1-13

CHUNG H, UP4-52, UP5-18

CHUNG J, MP10-10, MP15-5, MP18-20, MP21-18, MP22-8,

MP23-5, MP32-4, UP2-47, VS2-10, VS11-12

CHUNG K, MP6-13, MP14-3, MP21-18, UP3-53, UP6-16

CHUNG M, MP10-10, MP22-8

CHUNG S, MP15-5, MP32-4, UP6-51

ÇICEK M, MP24-2, VS1-13, VS14-10, VS15-6, VS17-3

CILENTO BG, MP26-5

CISU T, MP29-9

ÇITGEZ S, UP2-20, UP4-22, UP6-26, UP6-48

CITGEZ S, MP13-8, MP31-18

CLARK R, UP3-37

CLAROS OR, UP1-1, VS8-3

CLAYMAN RV, MP2-14, MP5-16, MP5-21, MP12-22, MP19-13, MP19-14, MP21-13, MP22-14, MP27-20,

MP31-8, UP1-19, UP1-27, UP1-52

CLEMENTI M, MP11-22, MP27-17, UP3-16,

UP3-28, UP3-58

CLEVELAND R, MP29-6

CLOUTIER J, MP24-21

CLOVIS S, MP11-18

COBLEY J, MP27-10

COCHETTI G, UP5-14

CODAZZI F, MP6-18

COKER C, MP13-11

COLEMAN J, MP30-19

COLICCHIA M, MP5-2, MP21-4

COLLIN N, MP7-8

COLOM S, MP7-11, UP4-46

COLOMBO R, MP22-1

CONE EB, MP33-4

CONNOR JA, MP18-6, MP23-3, MP25-20, UP2-7, UP5-30

CONNOR M, MP4-18, UP3-55

CONROY D, MP10-17

CONSIGLIERE D, MP22-13

CONSOLMAGNO H, MP22-3, MP25-22

CONSUELO C, UP6-49, VS11-7, VS11-9

COOGAN C, MP18-5

COOK J, UP3-42

COOPER JL, MP10-5

COOPER V, MP5-21, MP22-14, MP27-20, UP1-19

COPPENS E, MP24-18

CORBETT S, MP14-19

CORDEIRO A, MP8-4, UP4-21 
CORDES J, MP5-15, MP10-19, MP29-14

CORNELLA J, UP3-11

CORREA-LÓPEZ P, MP2-8

CORREIA J, UP5-53

CORSI P, MP21-11

CORTES RAYGOZA P, UP4-11

CORTES RAYGOZA PA, VS6-11, VS18-8

CORTIÑAS GONZALEZ J, MP3-11

COSCIONE A, MP19-3

COSER S, MP24-4, MP29-20

COŞKUN B, UP4-43

COSSU M, MP14-10, MP15-4

COSTA M, MP6-3

COSTA P, MP14-1, MP30-9

COSTANTINI M, MP1-7, MP16-13, MP16-17

COTTA B, UP5-4

COTTOM L, UP3-54

COWLEY A, MP2-2

CRACCO CM, UP3-3, UP3-4, VS3-6

CRIGGER C, UP3-40, VS6-3

CRISPEN P, MP4-14

CRIVELLI J, UP6-22

CROWLEY V, MP31-16

CRUZ, J.E.D.E.L.A., MP27-12

CUADRADO J, UP4-46

CUCE F, UP2-26

CUESTA-CAMUNAS B, MP15-6, MP16-7, UP2-4, VS7-11, VS18-7

CUI HW, MP5-5

CULPAN M, MP25-8

CUMARASAMY S, MP1-1, MP4-3, MP18-19, MP23-13, MP25-16, UP5-8

CUMPANAS A, MP19-4, UP1-22

CUMSKY J, UP3-11, UP5-27

CUÑÉ J, MP10-6

CUNITZ B, MP5-1, MP29-11

CURCIO L, MP16-11

CUSTER K, MP25-15

CUTNER A, MP14-15

CYNK M, UP3-32

\section{D}

DADONE C, MP32-2, MP32-10

DAGDEMIR A, VS6-2

DAGENAIS J, MP16-6, MP16-15, MP18-4, MP23-18, MP25-12, MP25-13, MP30-10, UP2-1, UP2-12, UP5-2

DAI JC, MP5-1, MP29-11, UP1-43

DAIZUMOTO K, MP33-13

DAL MORO F, MP5-2, MP21-4

DALMASSO E, MP32-2, MP32-10

DAMIEV A, MP30-21

DAMINESCU L, MP19-4, UP1-22

DANESHDOOST R, UP4-7

DANILOVIC A, MP13-9, MP13-10

DARABI MAHBOUB M, UP4-10

DARAWSHA A, UP1-20

DASGUPTA P, MP19-1, MP19-2, MP19-6, MP19-15, MP19-18, MP21-3, UP3-36, UP4-58, UP5-59

DAUDON M, MP13-16, MP24-21, UP1-24

DAUW CA, MP4-21, MP28-9

DAVALOS JG, MP12-20, MP26-2, MP32-17, VS1-6

DAVICIONI E, MP1-1

DAVID RA, MP3-2, UP1-2, UP5-55

DAVIES M, MP29-4
DAVIS L, MP10-20

DAWABA M, MP21-14

DAWIDEK M, UP2-54

DAWSON C, MP13-7

DAY AW, UP5-13

DE CASTRO ABREU A, VS7-1

DE CILLIS S, MP15-4, UP2-33, UP5-38

DE CONCILIO B, UP2-3

DE CONINCK V, MP2-5, MP5-4, MP5-6, MP5-11, MP5-14, MP5-20, MP10-2, MP12-3, MP13-16, MP18-10, MP19-4, MP24-5, MP27-5, UP1-21, UP1-24, UP5-16, VS1-1, VS1-2, VS1-4, VS1-10

DE DOMINICIS M, MP1-5, MP30-11

DE LA ROSETTE J, MP24-17

DE LORENZIS E, MP5-7, MP22-1, MP31-3, UP1-44, UP1-45, UP4-8, VS15-3

DE LUCA S, UP2-33, UP5-38

DE LUYK N, MP25-10, MP25-18

DE MARCHI D, MP24-20, VS1-12

DE PEDRO J, MP25-4, VS14-13

DE RUYSSCHER C, MP9-7

DE SOUSA MORAIS N, MP8-4, UP4-21

DE SOUZA DB, MP1-2, MP1-3

DE LA ROSETTE J, MP21-16

DE VINCENTIS L, MP23-20

DELONG J, MP22-12

DENAPOLI R, UP1-15

DEANE L, VS18-11

DECAESTECKER K, MP20-17, UP2-56

DEFIDIO L, MP1-5, MP30-11

DEGEN M, MP20-15, MP22-6, UP3-25

DEGIRMENCI E, MP32-16

DEINUM J, UP2-24

DEL FABBRO D, UP3-3, UP3-4, VS3-6

DEL POZO JIMÉNEZ G, MP16-20, MP25-3, MP25-4, MP33-10, UP5-3, VS14-13, VS16-14, VS17-5

DEL ZINGARO M, UP5-14

DELIVELIOTIS C, MP27-3

DELL'ORTO P, MP31-3

DELLIS A, MP27-3, UP6-4

DEMIR O, UP2-26

DEMIRDAG C, MP13-8, MP31-18, UP4-22, UP6-26, UP6-48

DEMIRDAG O, UP2-20

DEMIREL C, MP32-9

DEMIRKESEN O, VS10-1

DENES B, VS5-9

DENK J, UP6-11

DENSTEDT J, MP19-15

DERISAVIFARD S, MP7-20

DERVISHI A, MP11-10, UP5-39, VS18-13

DERWEESH I, MP18-21, MP23-1, MP23-6, UP5-4, VS8-8, VS8-9

DESAI A, MP8-2, MP8-3, MP21-7, MP31-12, MP32-7, UP1-23, UP4-54, UP4-55

DESAI M, MP7-6, MP18-2, MP31-19, MP31-22, UP3-43, UP6-52, VS12-1

DESAI MM, VS7-1

DESHMUKH C, MP7-6

DESHPANDE A, MP13-18

DEVANA SK, UP1-3, VS2-1

DEVASIA A, VS14-1

DHARIWAL R, UP3-30

DHOLARIA P, MP12-18, MP26-7 
DI GIANFRANCESCO L, MP1-4, UP1-8

DIPINA T, MP8-8, MP29-2

DIAH J, MP26-2

DIAMAND R, MP24-18

DÍAZ ÁLVAREZ J, VS2-2, VS15-9

DÍAZ ROMERO JM, MP3-11

DILEK M, VS13-1

DILL M, UP1-15

DIMOV A, VS1-3

DINCER B, MP17-22

DINDA A, MP1-13

DINH V, UP4-1

DIRI B, UP6-2

DIRI MA, MP3-21, UP6-2

DIRIE N, MP29-17

DIVAKARAN P, UP1-13

DIXON CA, MP21-19

DIXON CM, MP23-15, UP1-59

DODGE N, VS3-4

DOGANCA T, MP16-2, MP18-1, VS9-7

DOĞANCA T, MP6-11, MP20-10, UP1-48

DOGRA P, MP1-14

DOIZI SS, MP2-5, MP5-4, MP5-11, MP5-14, MP5-20, MP10-2, MP13-16, MP18-10, MP19-4, MP24-5, MP24-17, MP24-21, MP27-5, UP1-21, UP1-22, UP1-24, UP5-16, VS1-1, VS1-4, VS1-10

DOMBROVSKIY V, UP4-30

DOMINGUEZ BEAUTELL A, MP33-10

DOMINGUEZ-RODRIGUEZ J, MP2-8, MP12-1

DONALISIO DA SILVA R, MP27-8

DONG L, MP22-2

DOO S, MP1-17

DOPPALAPUDI S, MP4-3, MP18-6, MP23-3, MP23-13, MP25-20, UP1-35, UP2-7, UP5-30

DORE K, MP33-11

DOTAN Z, UP5-31

DOUMERC N, MP20-17, UP2-56

DOWN C, MP13-11, UP5-13

DOYLE H, MP8-11

DRAGOS LB, MP6-8, MP19-4, MP19-15, MP24-5, MP28-21, UP1-22

DREWA T, VS5-9

DRU C, UP4-49

DU K, MP16-12, MP21-7, MP25-7, UP1-23

DUAN H, MP7-10

DUAN X, UP1-25

DUBI-SOBOL A, UP6-37

DUBNANSKY A, MP10-17

DUDAROV A, MP30-20

DUGAN A, MP24-14, MP25-2

DUNMIRE B, MP5-1, MP29-11

DUPLISEA JJ, MP17-9

DURDIN TD, MP22-21, VS15-11

DUTTA R, MP23-2

DUVDEVANI M, UP1-46

DY G, MP14-8, MP14-9

DYMKOV I, VS13-9, VS14-4

DYMOV A, MP1-6, MP30-21, VS4-7

DYTRYCH P, UP2-10

E

ELGAMMAL M, MP30-4

EL-NAHAS A, MP9-19, MP28-5, MP29-21, UP4-36

EATON S, VS10-8
EBARA S, UP2-43

ECER G, VS5-1

EDAMURA K, UP2-43

EDDY B, MP4-10, MP20-2

EDDY BA, MP30-1

EDEN C, MP18-7

EDGARD L, UP4-17, UP4-18

EDWAN M, UP3-60

EDWARDS A, VS18-13

EDWARDS R, MP30-1

EFRATI S, UP1-31

EFTHIMIOU I, UP4-44

EHDAIE B, UP1-37

EILENDER B, MP9-17

EISNER B, MP12-16, MP26-20, MP28-22

EKER P, MP2-18, UP1-30

EKSI M, MP20-7, VS9-6

EL GHONIEMY M, MP21-1

EL SHAER W, MP11-15

EL TAYEB MM, MP22-21, MP24-10, MP33-15, UP3-29, UP4-56, UP5-44

EL YAZAMI O, VS2-7, VS8-11

EL SHEEMY M, MP21-1

ELSHEEMY MS, MP30-4

ELATREISY A, MP31-19

ELBAZ E, MP8-15

ELDIASTY IF, MP11-1, VS4-1

ELGAID Z, MP10-15

ELGHOBASHY S, UP1-9

EL-ASSMY A, MP9-14, UP3-60

EL-HASSAN M, MP4-6

EL-TATAWY H, MP33-14

ELHAGE O, MP11-18, MP22-5

ELHILALI M, MP3-3, MP24-15

ELISEU M, MP28-4, MP28-16

ELKASHEF AH, MP9-14, UP3-60

ELKASSABANY N, MP15-10, UP2-34

ELLIOT S, MP22-12

ELLIOTT D, MP3-20

ELLIOTT P, MP15-3

ELLIS J, VS2-9

ELMAHDY AM, MP11-4

ELMANSY H, UP3-7, UP3-8, VS3-2

ELMCHERQUI M, VS2-7, VS8-11

ELMEKRESH A, MP24-10, MP33-15, UP3-29

ELMENAIR AM, UP3-60

ELSALMY S, UP2-10, VS14-5, VS14-9

ELSAMRA S, VS18-4

ELSERAFY F, UP4-9

ELSHABRAWY M, MP9-14

ELSHAL A, MP9-19, MP11-1, UP4-36, VS4-1

ELSHAZLY M, MP29-21, UP4-9

ELSHERIF E, UP3-5

ELSOBKY E, MP2-6, MP31-11

ELTABEY N, VS4-1

ELTERMAN D, MP33-17, UP3-17

ELZAKZOKY A, UP4-9

EMILIANI E, MP19-4, MP24-17, VS1-9

EMRAN A, MP21-1

ENDERS FT, MP2-1

ENDICOTT R, UP1-23

ENDO F, VS9-8

ENDO T, MP20-20

ENDO Y, UP2-30 
ENE C, MP2-7, MP3-4, MP24-1, UP1-5, UP4-33, UP4-45, UP5-51

ENGANTI B, VS8-5

ENGELHART S, MP7-3

ENGLESBE M, MP28-10

ENIKEEV D, MP1-6, MP2-13, MP3-1, MP3-5, MP3-17, MP6-20, MP11-12, MP11-21, MP30-21, UP6-46, VS1-3, VS3-1, VS3-5, VS3-10, VS4-2, VS4-7

ENIKEEV M, MP14-17, VS9-12, VS12-10, VS17-10

ENOKIDA H, MP17-11, MP21-21

ERAIBEY M, MP4-10

ERAKY A, UP3-60

ERDEM S, MP4-4, MP4-5, MP8-21, MP17-22, MP24-9, MP32-16, UP1-36, UP4-3, UP6-29

ERGAKOV DV, MP12-3, MP23-19, VS1-1, VS1-2

ERICKSON B, MP24-8

ERIHAN IB, MP26-10

ERKAN E, MP9-20

ERLICH T, MP10-22

ERONINI N, MP31-9

ERTAS B, MP2-18, UP1-30

ERTÜZÜN T, MP1-8

ESEN B, MP6-2, MP33-18

ESHGHI M, MP11-16, MP12-6

ESLAHI A, MP9-4, MP12-5

ESPINO G, MP22-12

ESTRADE V, MP27-4

ESTRELLA R, MP22-12

ETEMADIAN M, MP26-22

ETO M, VS5-3

EUN DD, MP14-5, MP14-6, MP16-5, MP16-18, MP23-6, MP25-16, UP2-50, UP5-1, VS6-10, VS16-6,

VS16-10, VS16-13

EVANS PR, MP25-1

EWALD J, MP7-3

EZER M, VS13-10

\section{F}

FADALLAH M, MP9-14

FAERBER J, MP8-20

FAHMY N, MP24-15

FALAGARIO U, MP1-1, UP5-8

FAN Y, UP5-18, UP6-15

FANG J, VS2-4, VS12-2

FANG W, UP5-23

FANIMI OO, MP3-2

FANTA M, UP2-10

FARCAS M, MP7-19

FAREED K, MP16-6, MP18-4, UP5-2, VS2-12, VS3-11, VS18-5

FAROOQ S, VS8-2

FAROOQ Z, MP8-7

FARROKHYAR F, MP20-14

FATHELBAB T, MP12-12, MP24-7, MP27-6

FATHI A, MP3-14, MP9-6

FATHY A, MP11-15

FATIMA C, UP6-49, VS11-7, VS11-9

FAULDS K, MP31-16

FAURE WALKER N, MP22-5

FAVORITO LA, MP29-7

FAWZY A, MP27-6

FEDRIGON D, MP28-17

FEGHALI J, MP29-22

FEI X, MP26-14, MP26-15, UP4-57

FENG Y, MP11-8
FERGANY A, MP16-6, MP18-4, MP30-10, UP2-12, UP5-2

FERNANDEZ NOYOLA G, UP4-11, VS6-11, VS18-8

FERNÁNDEZ PASCUAL E, MP25-3, VS2-5, VS16-14

FERNÁNDEZ-CONCHA J, MP7-11, MP10-6, UP4-46

FERNANDO A, MP17-2, MP25-18

FERRANDINO M, MP10-20, MP27-18, UP1-55, UP6-1

FERREIRA C, MP14-1, MP30-9

FERREIRO CC, MP7-11, UP4-46

FERRIERO M, MP1-7, MP14-12, MP16-13, MP16-17,

MP30-7, UP1-56, UP2-3, VS7-1, VS7-4, VS9-9, VS10-5,

VS16-4, VS17-4, VS17-9

FERRIGNI R, UP5-27

FIEDLER M, MP5-3

FIGDOR A, UP6-58

FIGENSHAU R, MP16-12, MP21-7, MP23-12, MP25-7, MP32-7, UP1-23, UP4-54, UP4-55

FIGUEIREDO A, MP28-4, MP28-16

FIGUEIREDO F, UP3-4, VS3-6

FIGUEROA A, MP11-19, UP4-17, UP4-18

FILSON C, MP8-12

FINCH W, MP7-2, MP7-12, MP7-22, MP32-15

FINK A, MP33-4

FINKELBERG E, MP11-22, UP3-16

FIORI C, MP6-1, MP14-10, MP15-4, UP2-33, UP5-38

FISHER G, MP14-15

FLAMMIA G, VS14-6

FLETCHER J, MP4-2

FLORES-TAPIA J, UP6-14

FLOROFF C, MP17-14

FOKIN I, VS9-12, VS17-10

FONTANA M, MP5-7, MP22-1, MP31-3, UP1-44, UP1-45, UP4-8, VS15-3

FOOTE C, VS2-9

FORNARA P, MP20-17, UP2-56, UP5-17

FORSTER L, MP13-1, MP27-7, UP5-40

FOSSE EB, MP16-3

FOSSION L, MP23-7

FOSTER L, MP7-13, MP17-2

FOWLER S, MP7-2, MP7-12, MP7-22, MP32-15

FRAGA A, UP5-53, VS8-6

FRANCO M, MP6-3

FRENDL D, VS5-12

FREUND J, MP21-16

FRIED N, MP5-9

FRIEDLANDER J, MP31-4, UP6-8, VS2-9

FROEMMING A, UP3-19

FU B, MP17-8, MP20-18, UP2-29

FUCHS G, UP4-49

FUGITA OE, MP22-3, MP25-22, VS8-3

FUJIHARA A, MP16-16

FUJII T, VS17-1

FUJISAWA M, MP15-8, MP16-14, MP20-20

FUJITA K, UP1-57

FUJITA M, VS17-1

FUKAWA T, MP33-13

FUKUHARA S, UP1-57

FUKUMORI T, MP33-13

FUKUMOTO T, UP2-43

FUKUOKA K, UP2-35

FULLERTON S, MP11-16, MP12-6

FUNK JT, MP3-6, UP3-22

FURLAN A, MP22-15

FURUKAWA J, MP15-8, MP16-14, MP20-20

FURUMIDO J, UP5-28 
G

GAAS M, VS9-12, VS17-10

GABOARDI F, MP24-20, UP4-24, VS1-12

GADELMOULA M, UP5-17

GADZHIEV N, MP5-22, MP10-7

GAGNEUR J, UP5-27

GAHAN J, MP11-12, MP19-8

GALAL E, MP12-12, MP24-7, MP27-6

GALIANO M, MP23-14

GALIDA M, MP8-19

GALLANTE B, MP24-22, MP29-5, MP32-5, UP6-6, UP6-20

GALLETTO E, MP32-2, MP32-10

GALLIOLI A, MP5-7, MP22-1, MP31-3, UP1-44, UP1-45, UP4-8, VS15-3

GALLUCCI M, MP1-7, MP14-12, MP16-13, MP16-17, MP30-7, UP1-56, UP2-3, VS7-1, VS7-4, VS9-9, VS10-5, VS16-4, VS17-4, VS17-9

GAMAL ELDIN A, UP3-5

GAMAL W, UP4-34, UP4-47, UP4-48

GANESHAN B, MP5-5

GANPULE A, MP7-6, MP18-2, MP31-19, MP31-22, UP3-43, UP6-52, VS12-1

GAO R, UP4-41

GAO T, MP16-6, MP16-15, MP18-4, UP2-1

GAO X, MP3-10, MP5-10, UP6-42

GARAYEV A, MP15-2, MP26-19, UP2-32, UP4-26, UP5-49, VS6-9

GARCÍA CRIADO E, VS2-5

GARCIA RAMIREZ J, MP2-16

GARCIA A, UP1-1

GARG M, MP17-1

GARG V, MP31-22

GARGOLLO P, MP14-18, MP14-19, VS6-7

GARISTO J, MP16-6, MP16-15, MP18-4, MP23-18, MP25-12, MP25-13, MP30-10, UP2-1, UP2-12, UP2-45, UP5-2, VS5-10, VS8-10

GARROU D, MP14-10, MP15-4, UP2-33, UP5-38

GARZA-GANGEMI AM, MP2-8, MP12-1

GASIMOV K, UP6-29, VS7-7

GAUDARD E, VS5-2

GAUHAR V, MP27-21, UP6-18

GAUNAY G, MP7-20

GAUTAM G, MP20-12, MP30-17, VS7-9, VS17-6

GAYA J, MP21-11, MP21-12, VS15-8

GAZEL E, MP3-8, MP20-9, MP33-16, VS3-7, VS3-8, VS10-7, VS13-8, VS13-10

GEARMAN D, MP3-20, MP33-19, VS3-4

GEAVLETE B, MP2-7, MP3-4, MP24-1, MP29-1, UP1-5, UP4-33, UP4-39, UP4-45, UP5-51

GEAVLETE P, MP2-7, MP3-4, MP24-1, MP29-1, UP1-5, UP4-33, UP4-39, UP4-45, UP5-51

GENTILE B, MP6-4, MP23-20, MP30-12

GENTILE CR, UP4-15

GEORGESCU D, MP24-1, UP1-5, UP4-33, UP4-39, UP4-45, UP5-51

GERAGHTY R, MP6-8, MP7-14, UP5-58, UP6-38

GERIAL EL, MP17-15

GERKE T, UP5-41

GERMINALE F, VS14-6

GERVASIO-ORTIZ E, UP3-49

GHANEM S, MP11-18

GHANI KR, MP24-6, MP24-11, MP27-1, MP28-9, UP4-32, VS1-5, VS2-6

GHAZI A, MP19-5, VS8-2, VS10-3
GHAZIKHANLOU SANI K, UP4-7

GHAZWANI Y, MP13-19

GHICULETE D, MP7-19

GHIRALDI EM, MP31-4, UP6-8, VS2-9

GHOBRIAL FK, MP9-14, MP11-1

GHONAIMY AS, UP3-5

GHONIEMA W, MP21-1

GHOREIFI A, UP4-10

GHOUBASHI A, MP26-9

GHOUSE S, VS8-5

GIACOBBE A, VS14-6

GIEL D, MP14-19

GIL GARCIA J, MP2-16

GIL VILLA S, MP21-8

GILBERT B, MP22-4

GILBERT N, MP29-14

GILDERSLEEVE R, MP23-16, MP23-17

GILL IS, VS7-1

GILLETT WR, MP28-9

GILLING P, MP6-5

GILLISPIE G, UP6-45

GIMENO-COLLADO A, MP25-4, UP5-3

GINEPRI A, MP4-6

GIORDANO A, MP6-1, UP2-33, UP5-38

GIRARDO M, UP3-11

GIULIANELLI R, MP6-4, MP23-20, MP30-12

GIUSTI G, MP24-17, MP24-20, UP4-24, VS1-12

GKENTZIS A, MP19-9

GLAL A, MP21-14

GLASS J, MP7-13

GLEBER R, MP21-15

GLYBOCHKO P, MP2-13, MP3-1, MP3-5, MP3-17,

MP11-12, MP11-21, MP14-17, UP6-46, VS9-12,

VS12-10, VS17-10

GMITRO A, MP4-9

GODBOLE H, UP3-55, VS4-3

GOFRIT ON, UP1-46

GÖGER YE, VS1-7, VS5-1, VS17-7

GOH H, MP15-13, MP18-13, MP20-11, MP25-19, UP5-10

GOH Y, MP17-21, MP22-13

GÖKCE MI, MP12-2

GOLAFSHAR M, UP5-27

GOLD S, VS18-1

GOLDBERG S, UP1-46

GOLDMAN CC, MP4-17

GOLI V, VS5-9

GÖLLERT C, UP5-17

GOLOLOBOV G, VS4-7

GOLOMB D, MP31-5

GOMEZ F, MP11-19, UP4-17, UP4-18

GOMEZ-BLANCO J, MP19-21

GONÇALVES G, MP1-2, MP1-3

GONZÁLEZ DOMÍNGUEZ M, MP24-3

GONZALEZ M, VS13-11, VS14-2

GOODMAN J, MP28-3

GOODWIN J, MP28-7, MP28-8, UP3-51

GOONEWARDENE S, MP18-8

GOPAL N, VS6-8

GOPALAKRISHNA A, MP33-21, UP3-33, UP3-34

GORELOV D, MP5-22

GORIN M, MP5-13, MP24-19, MP32-21

GOUMAS IK, MP28-21

GOYAL A, MP28-18

GÖZEN A, MP5-3, MP19-2 
GRABILL K, MP29-3

GRAHAM S, MP8-16, MP13-1, MP27-7, MP31-10, UP1-54, VS12-3

GRAHN A, UP5-12

GRAJO J, MP4-14

GRANBERG C, MP14-18, MP14-19

GRAND D, MP8-9

GRANIER E, MP27-5

GRANIERI M, MP14-8, VS16-5

GRANJA I, MP31-8

GRECO F, MP31-9

GREEN J, MP8-16

GREEN M, MP4-1, MP4-2

GREENBERG J, MP18-15

GREGÓRIO B, MP1-2, MP1-3

GREGORY NJ, MP2-4

GRESTY H, VS12-3

GREY B, MP8-11

GRGIC T, MP9-16

GRIDLEY C, MP10-5

GRIFFIN SJ, MP9-2

GRIFFITHS L, MP7-20

GRIGGS R, MP31-4, UP6-8

GRILLS RJ, MP24-12, UP5-57

GRIMES N, UP5-11

GROHS E, UP1-27

GROOMS N, MP2-3

GROSS A, MP2-13, MP3-1, MP3-5, MP3-17, MP6-17, MP10-7, MP11-5, MP11-9, UP3-13, UP6-46

GRØTTA O, MP16-3

GRUCELA AL, VS15-4

GU H, MP1-10

GUAGLIANONE S, MP14-12, MP16-17, MP30-7, UP1-56, UP2-3, VS7-1, VS7-4, VS9-9, VS10-5, VS16-4, VS17-4, VS17-9

GUAN K, MP2-14

GUERRA R, MP22-3, MP25-22

GUERRERO-PUTZ M, UP6-14

GUEST K, UP3-32

GUIDOS P, MP24-8

GUIGGI P, UP5-14

GUILLOTREAU J, UP3-23

GUL A, MP9-12

GUL M, MP3-21, MP9-12, UP2-13, UP6-2

GUL Z, MP16-18, UP2-50

GULIEV B, MP14-2, MP24-13

GÜLPINAR B, MP12-2

GÜLPINAR O, VS10-1

GULSEN M, UP5-46, VS6-2, VS8-4, VS13-1, VS13-7, VS14-11

GUNERI C, MP20-9

GÜNSEREN K, MP24-2, VS1-13, VS14-10, VS15-6, VS17-3

GUO F, MP12-6, VS6-8

GUPTA A, MP1-1, MP18-19

GUPTA M, MP19-2, MP24-22, MP29-5, MP32-5, UP6-6, UP6-20

GUPTA N, MP11-13

GUPTA P, VS11-2, VS11-3, VS11-4, VS13-3, VS14-8

GURBANI CM, MP13-22, UP5-43

GURBUZ A, UP4-22

GURDAL M, UP2-26

GURNANI R, MP24-19

GUSEYNOV M, MP12-3, VS1-1, VS1-2

GUSTAFSON D, MP27-8
GUZEL O, MP17-7, MP24-4, MP29-20

GUZZO T, MP13-5

H

H.K. N, MP3-22

HA U, MP17-17, MP26-6

HA Y, MP15-5, MP32-4

HAAS CR, MP6-6, MP9-13, MP28-6, UP2-27

HABIBI K, MP11-14

HABUCHI T, MP25-5, UP2-46, VS8-12

HADDAD AQ, MP11-10, UP5-39

HADDAD M, MP18-10, UP5-16

HAFIZ AT, MP21-14

HAGA S, UP2-43

HAGHPANAH R, MP12-5

HAIFLER M, UP2-5

HAINES K, MP1-1, MP18-19

HAJIHA M, MP7-3, MP7-4, MP15-1, UP2-31, UP4-1

HAJIRAN A, UP3-40, VS6-3

HAKARIYA T, MP15-7

HALE G, VS18-1

HALEBLIAN G, MP31-2

HALL TL, MP24-6, MP24-11, MP27-1, UP4-32

HAM W, MP18-13, MP32-12, VS7-12

HAMASAKI T, UP2-30

HAMEED A, VS13-5

HAMILTON A, UP2-51

HAMILTON BD, MP25-1

HAMILTON Z, MP18-21, MP23-1, UP5-4

HAMMAM O, UP1-9

HAMPTON L, VS17-14

HAN C, MP25-7, MP31-12, UP1-23, UP2-47

HAN D, MP14-3, MP21-18, MP23-1, UP2-47, UP6-16, UP6-44, VS8-8

HAN J, MP10-10, MP22-8, UP3-47, VS2-10, VS11-12

HAN W, MP32-12, VS5-13, VS7-12, VS15-13

HANADA I, UP6-53

HANANO L, UP5-54

HANAOKA M, MP28-7, UP3-51

HANNA L, UP6-39

HANNA M, MP9-17

HANNEMANN AJ, UP4-49

HANSEN A, MP25-1

HANUS T, UP2-10

HARA R, VS17-1

HARA S, UP2-6, UP2-15

HARADA K, MP15-8, MP20-20

HARBIN A, VS16-13

HARDING R, MP7-8

HARDY L, MP5-9

HAROUN H, MP33-14

HARPER B, MP16-9

HARPER JD, MP5-1, MP29-11, UP1-43

HARRAZ A, MP26-9

HARRIS AM, MP24-14, MP25-2, MP28-7, MP28-8, UP3-48, UP3-51

HARTSELL L, VS16-11

HASAN A, MP24-16, UP6-27

HASAN N, MP24-17

HASAN R, UP1-47

HASANAT M, MP12-4

HASHEM A, MP9-14, MP9-19, MP11-1, UP3-60, VS4-1

HASHIMOTO H, MP9-18

HASIZUME M, VS5-3 
HASSAN ME, MP14-4, MP14-14

HATANO K, UP2-38

HATTORI K, MP23-10, VS9-8

HATTORI S, UP2-6, UP2-15

HAWARY A, UP3-42

HAYASHI T, UP2-30, UP2-35

HAYMANN J, MP10-2

HE Q, MP18-18, MP23-8, UP1-11, UP6-31, UP6-32

HEALY KA, MP27-9, MP31-17

HEBERT KJ, MP33-21, UP3-33, UP3-34

HECTORS S, MP1-1

HEILBRONN C, MP9-17

HEIMAN J, MP3-18, MP6-14, MP29-2, UP6-41,

VS1-11, VS3-3

HEINRICH C, MP7-4

HELMY T, MP21-14

HELO S, MP33-21, UP3-33, UP3-34

HEMAL AK, MP16-5, MP23-2, MP25-16, UP2-50, UP5-1, VS5-5, VS9-10

HEMAL S, MP16-15, UP2-12, UP5-2

HEMMANT J, MP9-21

HENDRY J, MP7-9, MP12-13, MP26-4

HENG C, MP27-21

HENRI P, VS5-2

HENRY M, UP4-50

HENSLE T, VS6-8

HENSLEY P, MP28-7, MP28-8, UP3-51

HERMIDA J, MP33-10, VS14-13

HERNANDEZ MENDEZ E, MP12-1, UP4-11,

VS6-11, VS18-8

HERRERA HERNANDEZ LP, MP2-1, UP1-14

HESSE DG, MP30-2, UP3-52

HIBBERT J, UP6-35

HIDAS G, UP1-46

HIEDA K, UP2-35

HIGASHIHARA H, UP1-57

HIGGINS MM, MP24-14

HIGUCHI M, MP19-6, MP19-11, UP3-36

HIGUERA T, MP3-7

HIKITA K, MP15-11, MP15-19, UP2-36, UP2-37, VS17-11

HINATA N, MP15-8, MP16-14, MP20-20

HIRAI K, UP2-48

HIRATA T, UP2-43

HIRAYAMA T, MP15-20, MP20-6, MP22-18

HIROKI I, MP13-17

HISASUE S, MP20-8

HITTELMAN A, MP14-19

HO RHA K, MP16-13

HO A, UP1-28

HO B, MP6-1

HO C, UP3-31

HO CW, UP3-42

HO H, UP3-31

HOANG K, MP17-13, VS13-2

HOCKENBERRY M, MP15-9, MP15-10, UP2-28, UP2-34, UP5-52

HODDINOTT P, MP10-1

HOE V, UP3-6

HOENIG D, MP7-20

HOFMANN M, MP5-16

HOKENSTAD E, VS6-12

HOLLAND B, MP11-13

HOLLER T, MP19-5
HOLLINGSWORTH JM, MP28-9, MP28-10

HOLMES DR, MP2-1, UP1-14

HOLMES R, MP2-20, MP2-21, MP10-21

HOLT SK, UP1-43

HONDA M, MP15-11, MP15-19, UP2-36, UP2-37, VS17-11

HONEY RD, MP7-19, VS16-1, VS16-2

HONG M, MP13-21, MP24-12

HONG S, MP12-11, MP13-22, MP16-21, MP17-17, MP18-14, MP26-6, MP30-22, VS7-12

HONG Y, MP18-12

HONGO F, MP16-16

HOOGENES J, MP19-7, MP20-14, MP33-11, UP3-37

HOPKINS M, UP3-48

HOPLAND A, MP16-3

HORA M, UP2-10

HORIE K, VS13-6

HORIE S, MP28-11

HORIGUCHI Y, UP3-1

HORN C, MP4-18

HORSFIELD C, UP5-42

HOSSAIN M, MP21-5, UP3-14

HOSSAIN T, MP12-4

HOSSEINI M, MP9-4, MP12-5

HOTTA K, MP19-6, MP19-11, UP3-36

HOU S, MP30-14, UP4-16

HOUMAN J, UP4-49

HOWARD KN, VS15-4

HSI R, MP31-6

HSU L, MP14-5

HSUEH T, MP17-20, VS11-10

HU R, UP6-7

HU W, MP4-20, MP5-18, MP21-9, MP21-10, MP26-21, MP33-22, UP4-27

HUA H, UP2-51

HUANG A, MP17-20

HUANG C, MP16-19, VS7-14

HUANG E, UP5-18

HUANG G, MP19-17

HUANG H, MP1-19, MP25-21

HUANG I, UP5-18

HUANG J, MP1-21, UP2-42, UP6-5, UP6-36

HUANG M, VS8-12

HUANG T, UP5-18

HUANG W, UP5-18, VS2-4, VS7-14

HUANG Y, MP22-2

HUAULMÉ A, VS5-3

HUBOSKY SG, MP27-2, VS5-11, VS6-5

HUGHES S, MP21-5, UP3-14

HUGHES W, MP16-8

HULSE L, MP17-14

HUMPHREYS M, UP3-11, UP5-27

HUMPHRIES M, MP28-22

HUNG F, MP16-19, VS11-11

HUNG S, UP6-15, UP6-54

HUPE MC, MP29-14

HUSEIN A, MP12-7

HUTCHINS G, MP4-2

HUYNH LM, MP15-12, MP22-19, UP5-19, UP5-20

HWA J, UP3-53

HWANG C, MP27-20

HWANG T, MP18-5

HYAMS ES, MP6-6, MP9-13, MP28-6, MP33-12, UP2-27

HYNES KA, MP3-6, UP3-22

HYUN J, UP3-53 


\section{I}

IACOBOAIE C, MP19-4, UP1-22

IAFRATE M, MP21-4

IBRAHIEM E, MP9-19

IBRAHIM AI, MP3-3, MP24-15, MP31-19

IBRAHIM E, VS4-1

IBRAHIM I, UP1-47

ICHIMARU N, MP17-16

IDRISSI KAITOUNI M, MP24-18

IEDA T, MP28-11

IGBOKWE MC, UP1-2

IINUMA K, VS13-6

IIO H, MP9-18

IKEDA T, MP15-20

ILGI M, MP32-9

IMAMURA R, MP17-16, UP1-57

IMOTO M, MP9-18

INALOO R, MP12-5

INCE Ü, MP1-8

INGELS A, MP23-14

INGEMI A, MP17-14

INGIMARSSON JP, UP4-15

INGLEBY H, UP1-50

INMAN B, MP6-19, MP28-19

INOUE K, MP30-3

INOUE S, UP2-35

INOUE T, MP25-5, UP2-46, UP2-48, VS8-12

INOUE Y, MP16-16

INSTON N, MP14-4, MP14-14

INTILLA O, UP5-26, VS12-8

IORGA M, MP11-16

IQBAL N, MP12-8, MP24-16, MP31-20, UP6-27

IRANI D, MP12-5

IRELAND J, MP30-1

IRIVING S, MP7-2

IRVING S, MP7-12, MP7-22, MP32-15

ISEDA T, MP9-18

ISHIDA R, MP23-10

ISHII D, MP20-6

ISHIKAWA S, UP4-59

ISHIMURA T, MP20-20

ISHITO N, MP9-18

ISOTANI S, MP28-11

ISRAEL G, MP4-12

ITESAKO T, MP17-11

ITO H, UP5-48

ITO Y, UP5-28

IWAMOTO H, MP15-19, UP2-36, UP2-37, VS17-11

IWAMURA M, MP15-20, MP20-6, MP22-18

\section{$\mathbf{J}$}

JABBOUR M, MP12-14

JABER J, MP14-21, UP2-5

JABER Y, MP17-9

JACKMAN S, MP8-5, MP23-7, UP6-11

JACOBSOHN K, MP23-6, VS9-4

JACOBSON K, MP7-8, UP1-17

JACOBY A, MP14-9

JAFAROV P, UP3-18

JAIKARANSINGH D, MP4-18

JAIN A, UP3-43

JAIN R, MP5-12, MP28-17

JAIN S, MP19-9

JAIPURIA J, MP17-1
JALALY J, MP25-7

JAMAL J, VS16-9

JAMES A, UP3-48

JAMES B, UP5-55

JAMES R, MP19-13, MP19-14

JAMES W, MP10-9

JAMIL I, MP12-8

JAMNADASS E, UP6-34

JAN H, VS12-11

JANARDANAN S, UP6-17, UP6-35

JANG G, MP13-3, UP1-7

JANG W, MP15-13, MP18-13, MP20-11, MP25-19, UP5-9, UP5-10, UP5-22

JANNIN P, VS5-3

JANSSEN M, UP2-56

JAREMKO G, UP5-12

JAUN J, MP30-1

JAVALI T, MP3-22

JAVIDIPARSIJANI S, VS18-11

JAVIER-DESLOGES JF, MP13-12, MP27-16, UP3-52

JAYARATNA I, MP1-1

JEH S, UP3-53

JENKINS L, MP10-5

JENNINGS B, MP24-12

JEON H, MP18-12, UP5-21

JEON S, MP18-12, MP22-20, UP2-47, UP5-21

JEON Y, MP6-12

JEONG B, MP18-12, UP2-47, UP5-21

JEONG C, MP13-3

JEONG H, MP13-15, MP29-15

JEONG S, UP4-52

JESSOP M, MP8-13

JHAGROO R, MP10-13, MP10-14

JHAVERI H, UP5-41

JI ZG, MP23-21

JIANG G, MP4-22, MP17-4, UP2-11

JIANG L, UP3-50

JIANG P, MP31-8

JIANG R, MP9-11

JIANG X, UP6-7

JIKUYA R, MP19-10, MP19-12

JIN D, UP4-1

JINDAL R, MP10-8

JOHN LM, MP2-15

JOHNIN K, MP14-20

JOHNSON B, MP8-17, MP8-18, MP11-12, MP19-8, MP23-11, MP27-13, UP1-34, UP1-38, UP6-22, VS5-6

JOHNSON K, MP13-12

JOHNSON M, MP4-1

JOHNSON S, MP2-15, VS9-4

JOHNSTON AW, MP9-11, MP28-1, MP33-4

JOHNSTON TJ, MP24-17

JOICE G, MP5-13, MP32-21

JONES A, MP4-6

JONES C, MP30-5

JONES GE, MP7-9, MP12-13, MP26-4

JONES P, MP6-8, MP7-14, MP9-3, MP27-19, MP32-8,

MP33-1, UP5-58, UP6-38

JONES R, MP21-5

JONGJITAREE K, VS1-8

JOO J, MP18-20

JORDAN J, MP1-1

JOSE A, UP6-49, VS11-7, VS11-9

JOSEPH A, UP4-50 
JOSEPH J, MP19-5, VS8-2, VS10-3

JOSEPH JP, MP18-9, VS16-7

JOSEPHSON D, UP4-49

JOSHI H, MP1-15, MP13-13, MP13-14

JOSHI P, MP32-22

JOSHI V, MP12-18, UP3-27

JOUNG J, MP25-6

JUNG E, MP8-9, MP32-6

JUNG H, MP6-13, MP31-15, MP32-12, UP4-42

JUNG HU, MP2-10, MP2-11

JUNG J, MP13-15, MP29-15, MP33-7, VS5-13

JUNG N, VS15-15

JUNG S, UP4-52

JUNG W, MP15-5, MP32-4

\section{$\mathbf{K}$}

KACHRILAS S, UP5-13

KACHROO N, VS7-3

KADASNE R, MP31-11

KADIHASANOGLU M, MP9-20

KADNER G, MP6-1

KAFKA I, MP14-21, UP2-5

KAGEYAMA S, MP14-20

KAHAN A, MP18-5

KAHN L, MP5-12

KAIFU M, VS17-1

KAILAVASAN M, UP4-50

KAJIKAWA K, MP29-13

KAKIMOTO K, UP2-38

KALE NC, VS4-3

KALER KS, MP5-21, MP12-22, MP19-13, MP19-14, MP22-14, MP27-20, UP1-19, UP1-27, UP1-52

KALLAPPAN S, VS6-4, VS6-6, VS14-3

KALLIDONIS P, MP3-9, MP21-2, UP6-30

KAM S, UP3-53

KAMAL N, UP4-1

KAMEOKA H, UP2-14

KAMPHUIS G, MP2-6, MP21-16, MP28-21

KANAO K, MP29-13

KANAYAMA H, MP33-13

KANDA S, MP25-5, UP2-46, VS8-12

KANDASWAMY GV, MP28-12, UP5-55

KANDASAMY G, UP5-40

KANDEEL W, MP11-15

KANEKO G, UP2-6, UP2-15

KANG M, UP5-21

KANG S, MP17-17, MP25-6, MP26-6

KANG T, UP4-52

KAO W, MP6-9

KAOUK J, MP16-6, MP16-15, MP18-4, MP23-18, MP25-12, MP25-13, MP30-10, UP2-1, UP2-12, UP2-45, UP5-2, VS5-10, VS8-10, VS9-5

KAPLAN A, MP27-9

KAPLAN JR, VS16-6, VS16-13

KAPLAN S, MP11-7

KAR A, MP6-10

KARA C, UP6-29

KARAKIEWICZ P, MP30-6

KARIM SULAIMAN S, UP6-39

KARIM T, MP12-4

KARSIYAKALI N, MP9-20

KARTAL I, VS6-2

KARTAL M, MP4-4

KARTALAS GOUMAS I, MP14-11, VS15-1
KARTHIKEYAN V, UP6-10

KASARANENI P, MP3-22

KASRAEIAN A, UP1-39

KATA S, UP1-47

KATAOKA M, UP2-14

KATO M, MP30-15

KATO T, MP17-16, UP1-57, VS13-6

KATZ A, MP23-16, MP23-17

KATZ J, UP2-51

KAUSHAL S, MP1-13

KAWADA T, MP9-18

KAWAKITA M, MP30-3

KAWAMOTO B, MP15-11

KAWAMURA K, UP2-39

KAWAMURA M, MP17-16

KAWAMURA Y, UP3-21

KAWAN F, UP5-17

KAWANO P, MP22-3, MP25-22

KAWASHIMA A, UP1-57, UP5-27

KAWAUCHI A, MP14-20, VS12-12

KAYA E, MP3-8, MP20-9, MP33-16, VS3-7, VS3-8, VS10-7, VS13-8, VS13-10

KAYES O, MP28-13, MP28-14, MP28-15

KAYGISIZ O, MP24-2, UP4-43

KAZAN Ö, MP25-8

KEEHN A, MP14-5, MP14-6, VS6-10, VS16-10

KEEL CE, VS15-12

KEELEY F, MP7-8, MP13-17, UP5-48

KELLER EX, MP2-5, MP5-4, MP5-6, MP5-11, MP5-14, MP5-20, MP10-2, MP12-3, MP13-16, MP18-10, MP19-4, MP24-5, MP27-5, UP1-21, UP1-24, UP5-16, VS1-1,

VS1-2, VS1-4, VS1-10

KELLER H, MP6-22

KELLER M, MP8-2, MP8-3

KELLNER D, UP3-20

KELLY N, MP7-13

KENETT R, UP1-31

KENNEY PA, MP13-12

KEOGHANE S, MP7-5

KERFOOT B, MP33-4

KERR L, MP7-9, MP12-13, MP26-4

KESAVAN N, UP3-2

KESKIN M, MP1-8, MP6-11, MP20-10, UP1-48

KESKIN S, MP16-2, MP18-1

KHALAF I, MP31-19

KHALID M, MP24-16

KHAN F, MP17-19, UP3-14

KHAN MS, MP19-6, MP19-18, UP3-36

KHAN N, MP1-13

KHAN NS, UP5-47

KHAN S, MP19-1, MP19-2, MP19-15, MP21-3, MP30-8, UP4-58, UP5-47, UP5-59

KHANNA A, VS16-12

KHONG B, UP1-17

KIKUCHI H, MP19-6, MP19-11, UP3-36, UP4-59, UP5-28

KIKUGAWA T, UP2-43

KILIÇCARSLAN H, MP24-2, UP4-43, VS1-13

KILINÇ M, VS1-7

KILINC M, MP13-6

KIM B, MP15-5, MP32-4

KIM C, MP6-13

KIM D, MP6-12, MP31-15, MP32-12

KIM EH, MP4-7, MP4-8, MP18-11, MP23-12, MP25-7

KIM F, MP27-8 
KIM H, MP3-16, MP10-4, MP15-5, MP26-3, MP32-4, UP4-13

KIM J, MP1-17, MP8-20, MP14-3, MP15-9, MP16-21, MP31-15, UP2-28, UP2-47, UP3-38, UP5-9, UP5-22, UP5-52, UP6-16, VS2-10, VS11-12

KIM K, MP1-17, MP6-12, MP6-13, MP12-11, MP26-6, MP33-7, UP3-37, UP3-47, UP6-16

KIM L, VS13-5

KIM M, UP4-52

KIM S, MP6-12, MP12-11, MP17-17, MP18-20, MP20-5, MP23-5, MP26-6, MP31-13, UP1-58, UP5-34, VS2-10, VS11-12

KIM T, MP6-13, MP15-5, MP18-12, MP25-6, MP32-4

KIM Y, MP1-17

KIMURA G, UP2-30

KIMURA Y, MP15-11, MP15-19, UP2-36, UP2-37, VS17-11

KINOSHITA H, MP19-19

KIRECCI S, MP32-9, UP3-18

KISHIDA T, UP5-35

KISLYAKOV D, MP1-6, MP30-21, VS4-7

KITAKAZE H, MP15-18, UP2-19

KITAMURA K, MP28-11

KITAYAMA S, MP29-19

KIUCHI H, UP1-57

KIZILAY F, UP6-9

KLAASSEN Z, MP16-8, MP20-3

KLEIN J, MP5-3

KLEINCLAUSS F, MP24-21, MP27-4

KLEINGUETL C, MP22-21, UP3-29, UP5-44

KLEINMANN A, UP6-59

KLEINMANN N, MP10-22, UP4-51, UP5-31, UP6-59

KLIMOV R, VS1-3

KLOPFER M, MP27-20

KLØW N, MP16-3

KNAUER CJ, MP4-3, MP23-13, UP1-35

KNIGHT J, MP2-20, MP2-21, MP10-21

KNOEDLER J, MP13-20

KNOLL P, MP11-10

KNOLL T, MP19-2, MP24-17

KNUDSEN BE, MP5-19, MP10-5, MP26-18, MP27-1, MP27-14, MP28-22

KO K, MP3-16, UP4-13

KO Y, MP15-12, UP4-42

KOBATAKE K, MP30-15

KOBAYASHI H, MP23-10

KOBAYASHI I, MP29-13

KOBAYASHI K, MP14-20

KOBAYASHI M, UP2-14

KOBAYASHI R, MP19-12

KOBAYASHI S, VS5-3

KOBAYASHI Y, MP15-16, MP25-9, MP25-11, MP30-16, UP2-22, UP2-39, UP6-55

KOCAASLAN R, MP26-10

KOCAK T, MP8-21, MP24-9, MP32-16, UP6-29

KOCH G, MP4-17, MP10-12

KOCH M, MP4-1, MP4-2

KOCHER N, MP13-20

KOCHEROV S, MP14-21, UP2-5

KOCHIEV D, MP30-20

KOGA S, UP3-1

KOH C, MP19-17

KOH D, MP15-13, MP18-13, MP20-11, MP25-19, UP5-10

KOHLER T, MP33-21, UP3-33, UP3-34

KOHNO Y, MP29-19

KOJIMA Y, UP2-14
KOMMU SS, MP4-10, MP20-2, MP30-1

KOMYAKOV B, MP14-2, MP24-13

KON M, UP4-59

KONDAKINDI P, VS8-5

KONDO H, UP2-6, UP2-15

KONDO Y, UP2-30

KONDRASHINA A, VS15-10

KONNO S, MP11-16

KOO K, MP3-16, MP18-14

KOO V, MP13-18

KORD E, UP1-31

KORDAN Y, MP33-8, VS15-6

KORETS R, MP12-16

KORKES F, VS8-3

KORKMAZ H, UP1-48

KOROLEV D, VS9-12, VS17-10

KORTEN M, UP3-13

KOSIOREK H, UP5-27

KOSTER H, MP18-6, MP23-3, MP25-20, UP2-7, UP5-30

KOSTOPOULOS A, MP13-11

KOTB A, UP3-7, UP3-8, VS3-2

KOTSIRIS D, MP3-9, MP21-2, UP6-30

KOTT O, MP8-9, MP32-6

KOURMPETIS V, MP29-10, MP32-18

KOUTSOS C, UP3-45

KOVALENKO A, MP5-6

KOWALSKI F, VS5-9

KOYASU H, MP28-11

KRAHN M, MP33-17

KRAMBECK AE, MP2-1, MP3-18, MP4-21, MP6-14, MP10-9, MP28-22, MP29-2, UP1-14, UP6-41, VS1-11, VS3-3

KRAMER MW, MP10-19, MP29-14

KRANE L, MP18-15

KRAUSE R, UP4-1

KREIDER W, MP5-1

KRISHNAMURTHI V, MP4-11

KRISHNAN J, MP19-9

KROON B, UP2-57

KUBOTA M, MP30-3

KUBOTA Y, MP19-10

KUCHERIA R, MP28-18

KUEBKER J, MP11-2

KULKARNI M, MP22-5

KULKARNI R, UP6-17

KUM FE, MP19-18, MP22-5, UP3-30

KUMAR A, VS11-2, VS11-3, VS11-4, VS13-3, VS14-8

KUMAR K, MP27-11, UP3-46, UP3-57

KUMAR N, VS11-2, VS11-3, VS11-4, VS13-3, VS14-8

KUMAR S, MP10-8, UP1-3, VS2-1, VS8-7, VS11-2, VS11-3,

VS11-4, VS13-3, VS14-8, VS16-12

KUNIT T, MP19-2

KUO J, UP5-18

KURA M, MP2-6

KURAL A, MP1-8, MP6-11, MP16-2, MP18-1, MP20-10, UP1-48, VS7-7, VS9-7

KURASHIMA Y, MP19-6, MP19-11, UP3-36

KURIBAYASHI S, MP15-18, UP2-19

KURTI M, VS14-6

KURTZ MP, MP26-5

KUSTERS B, UP2-24

KUSUHARA Y, MP33-13

KUSUMA MV, MP18-7, MP30-5, UP2-18, UP2-53

KUTIKOV A, MP16-13

KUTSAL C, MP32-9 
KWOK J, MP16-10

KWON D, UP4-52

KWON T, MP15-5, MP32-4, UP1-42

KYNASTON H, MP28-12

$\mathbf{L}$

L'ESPERANCE J, MP17-5

LAGUNA PES M, MP23-7

LAHME S, MP19-15

LAI B, MP30-14

LAI D, UP4-14

LAI Y, MP17-20

LALL C, UP1-52

LAM V, VS13-5

LAMA D, UP1-52

LAMA-TAMANG T, MP18-6, MP23-3, MP25-20, UP2-7, UP5-30

LAMB B, MP8-16

LAMOSHI A, MP14-16, MP23-4

LAN Y, UP1-25

LANCASHIRE M, MP13-18

LANDAU E, UP1-46

LANDMAN J, MP2-14, MP5-16, MP5-21, MP12-22, MP19-13, MP19-14, MP21-13, MP22-14, MP23-7, MP27-20, MP31-8, UP1-19, UP1-27, UP1-52, UP5-29

LANDRIGAN-OSSAR M, MP26-5

LANGE B, MP5-15

LANGE D, MP2-9, MP2-17, UP1-26, UP1-29

LANGENHUIJSEN H, UP2-24

LANTZ A, MP19-2

LAOYE A, UP1-2

LAPIN S, MP31-21

LARCHER A, MP16-17

LARGE T, MP3-18, MP6-14, MP10-9, MP29-2, UP6-41, VS1-11, VS3-3

LARNER T, MP23-9, MP30-1

LARSON K, MP2-14

LATCHAMSETTY K, MP18-5

LAU B, MP9-15

LAU H, VS13-5

LAU W, MP1-19, UP5-25

LAUKHTINA E, MP11-21, VS17-10

LAURIDSEN J, MP16-3

LAVAN L, MP31-14

LAVIGUEUR-BLOUIN H, UP1-49

LAWRENTSCHUK N, MP29-16

LAY A, MP8-12, MP8-17, VS16-11

LAYMON M, MP9-14

LAZAROU L, UP6-4

LAZAROVICH AY, UP4-51

LE ROUX P, UP2-23

LE SUEUR A, MP23-16, MP23-17

LE H, MP21-15, VS6-1

LEAL M, UP4-18

LEAL RA, MP11-19

LEAPMAN M, UP3-52

LEAVITT DA, MP7-20, MP9-17, MP27-9, MP28-9

LEBEDEV D, MP31-21

LECHEVALLIER E, MP23-7

LEE A, MP31-4, UP6-8

LEE BR, MP1-10, MP1-11, MP4-9, UP2-51

LEE C, MP6-12, UP3-47, UP3-53

LEE D, MP3-16, MP10-4, MP15-9, MP15-10, MP26-3, MP31-6, UP2-28, UP2-34, UP5-52
LEE H, MP3-19, MP16-21, MP18-12, MP30-22, UP3-38, UP5-21, VS7-12, VS15-13

LEE J, MP3-13, MP3-19, MP7-19, MP10-4, MP12-11, MP15-5, MP15-13, MP17-17, MP18-13, MP20-11, MP25-19, MP26-3, MP26-6, MP31-15, MP32-4, MP32-12, UP1-10, UP5-10

LEE K, MP12-11, MP17-17, MP26-6

LEE L, UP2-21

LEE R, VS16-10

LEE S, MP10-4, MP10-10, MP16-21, MP18-12, MP22-8, MP22-20, MP26-3, MP30-22, UP2-51, UP3-53, UP4-13, UP5-11, UP5-22

LEE T, UP1-19

LEE W, UP5-57

LEE Z, MP14-5, MP14-6, VS6-10, VS16-6, VS16-13

LEGEMATE JD, MP21-16

LEGRAND F, MP24-18

LEHMAN K, MP13-20

LEIBOVICH BC, MP18-9, VS15-2

LEIBOVICI D, MP8-1, MP8-15, UP6-13, UP6-37

LEKAREV V, MP30-21, VS1-3, VS4-7

LEMOS GC, UP1-1, VS8-3

LEONARD J, MP31-16

LEONG J, VS5-11

LEOW JJ, UP5-43

LEROY A, VS5-2

ŁESIÓW M, VS6-14

ŁESIÓW R, VS6-14

LESLIE S, VS13-5

LETENDRE J, MP24-21

LETRAN JL, MP1-18, MP15-17

LEUNG A, MP8-12

LEUNG CP, MP30-2

LEUNG S, MP9-15

LEVY N, MP29-10

LEVY O, MP5-1

LEWIS SC, MP1-1, MP4-3, MP18-3, MP18-19, MP23-13, UP1-35, UP5-6

LEZREK M, VS2-7, VS8-11

LI A, UP5-23

LI C, MP9-22, MP29-17

LI E, MP20-4

LI G, MP28-2, MP31-17, MP33-12

LI H, MP3-10, MP14-13, MP18-18, MP23-21, UP1-11, UP4-40, UP4-41, UP6-31, UP6-32

LI J, MP1-12, MP2-19, MP4-13, MP4-20, MP5-18, MP9-10, MP16-9, MP21-9, MP21-10, MP25-12, MP26-21, MP27-18, MP29-12, MP30-14, MP33-22, UP4-16, UP4-27, UP5-24, UP6-24, VS10-8

LI L, MP5-10, UP6-42

LI P, VS15-5

LI Q, MP2-2

LI R, MP17-9

LI S, MP8-19, MP14-3, VS11-10

LI W, UP5-23

LI X, MP1-21, MP8-22, UP4-14

LIANG C, MP1-12

LIANG L, UP5-24

LIANG Y, UP2-42

LIAO B, MP14-13, UP4-40

LIATSIKOS E, MP3-9, MP21-2, UP6-30

LIE K, UP3-2

LIEN C, MP16-19, VS11-11

LIESBETH D, MP20-17, UP2-56

LIESKE JC, MP2-1, UP1-14 
LIFSHITZ D, MP31-5, UP1-20

LIGGITT H, MP5-1

LILDAL SK, MP2-10, MP2-11

LILOV M, MP32-14

LIM H, UP3-2

LIM J, UP3-2, UP5-56

LIM K, UP4-16

LIM S, MP32-21

LIM Y, UP2-21

LIMA E, MP8-4, UP4-21

LIN A, UP5-18

LIN C, MP19-13, MP19-14, UP1-52, UP3-9, UP5-18, UP5-29

LIN G, MP13-5

LIN J, VS7-13

LIN T, UP5-18

LIN V, UP2-25, VS9-3

LIN Y, MP1-21, UP2-42, VS12-11

LINARES E, MP18-21

LINDEBLAD M, MP2-2

LINDER B, MP3-20, VS6-12

LINDNER U, MP8-1, MP8-15, UP6-13, UP6-37

LINDSAY J, UP2-53

LINGEMAN JE, MP3-18, MP4-21, VS3-3

LINK R, MP19-16

LIOURDI D, MP3-9

LIOUTIS M, UP2-1

LIPKIN ME, MP9-11, MP10-20, MP27-18, MP28-1, MP29-12, UP1-55, UP6-1

LISETH O, UP6-33

LIU B, MP1-12

LIU H, MP2-2

LIU JC, MP14-6, VS16-13

LIU M, MP5-10, MP11-6, UP6-42

LIU N, MP30-19

LIU S, MP23-8, UP5-23

LIU Y, MP4-20, MP21-9, UP6-31, UP6-32

LIU Z, MP29-11

LO C, VS17-2

LO K, UP4-16

LOBO N, MP4-10, MP13-11, MP20-2

LOEB A, VS3-12

LOH A, UP3-56

LOKMANE E, VS8-11

LOMBARDO R, MP6-3, MP30-12

LONGO F, MP5-7, MP22-1, MP31-3, UP1-44, UP1-45, UP4-8, VS15-3

LÓPEZ DÍEZ M, VS2-2, VS15-9

LOPEZ MAGUEY RP, VS6-11, VS18-8

LOPEZ SILVESTRE J, MP21-8

LÓPEZ-ACÓN J, VS2-8, VS15-14

LOPEZ-HUERTAS H, MP16-7, UP2-4

LOPEZ-SAMANO V, UP3-49

LORENTZ A, VS16-11

LORUSSO V, MP31-3

LOUNICI M, UP4-38

LOVALLO G, MP18-6, MP22-6, MP23-3, MP25-20, UP2-7, UP5-30, VS6-8, VS6-13, VS7-5, VS18-6

LOVEGROVE C, MP4-18, MP19-15

LOVETT M, UP2-51

LOWRY P, MP24-10, MP33-15, UP4-56, VS15-11

LTEIF E, MP12-14

LU C, MP20-3

LU DD, VS10-3

LU H, UP5-23
LU S, UP5-18

LU Y, MP29-17, UP5-25

LUBIN M, MP16-18, MP18-16

LUDVIGSON AE, UP4-15

LUDWIG W, MP5-13, MP32-21

LUIS ANTONIO R, UP6-49

LUKE P, UP2-54

LUMEN N, MP6-1

LUO H, MP18-17

LUPERTO E, VS14-6

LUPTAK J, UP6-23

LUU T, MP18-6, MP23-3, MP25-20, UP2-7, UP5-30

LUZZAGO S, MP22-1, MP31-3, UP1-45, UP4-8, VS15-3

LWIN AA, MP3-6, UP3-22

LYNCH T, MP31-16

LYNN N, UP3-56

M

M. SAMI AK, UP1-4

MACDOUGALL RD, MP26-5

MACCHIONE N, MP19-1, MP21-3

MACCONAGHY B, UP4-32

MACEK P, UP2-10

MACHEN G, MP22-21

MACIOLEK KA, MP10-11

MADDONNI S, UP5-14

MADDOX MM, VS16-3

MADEC F, MP12-21

MADI R, MP15-14, MP16-8, MP16-9, MP16-22, MP20-3

MAEKAWA Y, VS13-6

MAGHSOUDI R, MP26-22

MAHAJAN A, UP4-29

MAHESHWARI R, MP18-2, MP31-22, UP3-43

MAHMALJI W, MP27-10

MAHON J, MP6-15, MP11-13

MAILLOUX-SALINAS P, MP2-8

MAK S, UP4-16

MAKITA N, MP30-3

MAKIYAMA K, MP16-1, MP19-10, MP19-12

MALAGÒ G, UP1-44, UP1-45

MALCOLM J, MP17-14

MALDE S, UP5-47

MALDONADO-ALCARAZ E, UP3-49

MALIHA S, MP14-9

MALIYIL J, MP22-5

MALM C, UP5-12

MALTHOUSE T, MP13-11, UP5-13

MAMODE N, MP22-7

MANCHA E, MP19-21

MANDAL AK, UP1-3, VS2-1

MANDAVA S, MP18-15

MANDRON E, MP30-9

MANECKSHA RP, MP31-16

MANES C, UP1-26

MANFREDI M, MP14-10, MP15-4, UP2-33, UP3-28, UP3-58, UP5-38

MANGAT R, MP27-21, UP6-18

MANGHAT P, MP10-15

MANICKAM R, VS6-4, VS6-6, VS14-3

MANIT A, MP6-1

MANN R, UP2-24

MANSOUR A, MP9-19, UP4-36

MANTICA G, MP24-20, UP4-24, VS1-12

MANZO BO, MP11-19, MP26-20, UP4-17, UP4-18 
MANZO G, UP4-17, UP4-18

MARCHALIK D, MP4-17

MARCHINI G, MP13-9, MP13-10

MARCHIONI M, MP30-6

MARCONI L, MP25-18

MARCOS-MARÍN D, MP25-3

MARGULIS V, MP11-12

MARI A, MP16-13

MARKOLLARI V, MP8-2, MP8-3

MARKOPOULOS T, UP6-4

MARQUES V, MP28-4, MP28-16

MARQUES-PINTO A, VS8-6

MARSH BM, MP10-3

MARSH H, MP19-15

MARSHALL M, MP17-5

MARTA G, UP6-49, VS11-7, VS11-9

MARTIN S, MP3-11

MARTIN TV, MP30-2

MARTINEZ ARROYO C, MP7-21, MP12-1, UP4-11, VS6-11, VS18-8

MARTÍNEZ SAPIÑA-LLANAS I, VS15-9

MARTÍNEZ VÁZQUEZ A, VS15-9

MARTINI A, MP1-1, MP18-19, MP25-16, UP5-8, VS10-6, VS18-9

MARTINS M, MP6-18

MARTIROSYAN G, VS12-4

MARTIS SM, MP19-4, UP1-22

MARTOV AG, MP12-3, MP23-19, VS1-1, VS1-2

MARUCCIA S, MP11-22

MARUYAMA S, UP5-28

MARUYAMA Y, MP15-16, MP25-9, MP25-11, UP2-22, UP2-39, UP6-55

MASKAL S, MP5-12, MP28-17

MASTAI Y, UP1-20

MASTROIANNI R, VS14-6

MASUCCI L, MP33-17

MATLAGA B, MP5-13, MP24-19, MP27-9, MP28-22, MP32-21

MATSUBARA A, UP2-35

MATSUDA T, MP19-19

MATSUMOTO ED, MP19-7, MP33-11, UP3-37

MATSUMOTO K, MP15-20, MP20-6

MATSUMOTO R, MP19-6, MP19-11, UP3-36, UP4-59, UP5-28

MATSUOKA Y, MP15-18, UP2-19

MATSUSHITA K, VS9-8

MATSUSHITA M, MP15-18

MATSUZAWA I, UP2-30

MATZ E, MP6-19

MAUGERI O, MP32-2, MP32-10

MAURER T, MP18-21

MAURI G, MP6-18

MAVUDURU RS, UP1-3, VS2-1

MAXWELL AD, MP5-1, UP4-32

MAYNARD W, MP7-18, MP32-20

MAYOR NN, MP32-19

MAZZON G, MP14-7

MAZZONE E, MP30-6

MAZZUCCHI E, MP13-9, MP13-10, MP26-20

MCCAHY P, MP13-21

MCCOY JA, VS16-3

MCGILL A, MP15-9, MP15-10, UP2-28, UP2-34, UP5-52

MCKAY A, UP5-11

MCMAHON GC, UP3-15
MCPHEE A, UP3-54

MCVARY KT, MP6-15, MP11-13, UP3-10

MCWILLIAMS K, MP15-9, MP15-10, UP2-28

MEARINI E, UP5-14

MEDENWALD RC, MP2-15

MEDIAGO M, MP32-2, MP32-10

MEHROTRA S, MP17-6

MEHTA A, MP8-12, MP12-18, UP3-27

MEHTA RA, MP2-1, UP1-14

MEHTA S, MP2-6

MELNYK R, VS8-2

MELO P, MP26-20

MÉNARD J, MP30-9

MENDEZ-PROBST C, MP2-8, MP12-1, UP6-14

MENDIOLA DA, MP17-3, MP17-15

MENDOZA JS, MP15-17

MENG Y, MP18-11

MENG X, VS15-5

MENON A, MP4-6

MENSAH E, UP1-49

MENZIES T, MP27-7

MERCADER C, MP6-3

MERCHANT M, MP15-3

MERSEBURGER AS, MP10-19, MP29-14

MERT A, UP4-43

MESHKI I, MP26-22

MESSER J, MP11-10, VS18-13

METE U, VS2-1

METRO MJ, MP14-6, VS16-6, VS16-13

MICHAEL P, UP3-48

MIERNIK A, MP22-9

MIEST TS, MP18-9

MIKHAIL D, UP2-54

MIKHAIL M, MP7-15, MP14-7, MP14-15

MIKI D, UP2-48

MIKKILINENI N, MP31-17, MP33-12

MILK J, MP5-12

MILLAN F, VS1-9

MILLER A, MP2-9, MP2-12

MILLER AR, VS12-6, VS16-7, VS16-8

MILLER NL, MP6-19, MP11-2, MP28-19, MP28-22

MIMA M, UP4-1

MIMATA H, UP2-48, UP2-49

MIN K, MP32-4

MINERVINI A, MP16-13, MP23-6

MINISOLA F, MP14-12, MP16-17, MP30-7, UP1-56, UP2-3,

VS7-1, VS7-4, VS9-9, VS10-5, VS16-4, VS17-4, VS17-9

MIR M, MP18-21

MIRABILE G, MP6-4, MP23-20, MP30-12

MIRCIULESCU V, UP5-51

MIRZAZADEH M, UP6-45

MISHIMA T, MP19-19

MISHRA D, MP7-1

MISRAI V, UP3-23

MISURACA L, MP14-12, MP16-17, MP30-7, UP1-56, UP2-3,

VS7-1, VS7-4, VS9-9, VS10-5, VS16-4, VS17-4, VS17-9

MITA K, MP30-15

MITHAL P, VS2-7, VS8-11

MITSUI Y, MP15-16, MP20-8, MP25-9, MP25-11, UP2-22,

UP2-39, UP6-55

MIURA N, UP2-43

MIYAGAWA Y, MP15-18, UP2-19

MIYAJI Y, MP9-18, VS17-1

MIYAJIMA A, UP3-21 
MIYATA H, MP19-6, MP19-11, UP3-36, UP5-28

MIYATA Y, MP15-7

MIYAUCHI Y, UP2-43

MIZUTANI K, VS13-6

MMDOUH A, UP4-34, UP4-47, UP4-48

MOCHIZUKU H, MP30-15

MODAI J, MP8-1, MP8-15, UP6-13, UP6-37

MOGHUL M, MP7-16, MP7-17, MP28-18, UP3-55, UP4-2, UP4-19, UP4-20

MOHAGHEGH P. S, UP1-50

MOHAMED N, MP4-18

MOHAMED O, MP3-14, MP9-6

MOHAMMED A, MP17-19

MOHAMMED B, UP1-17

MOHAMMED N, MP20-17, UP2-56, UP5-17

MOHAPATRA A, MP22-17, MP30-19, MP33-6, UP3-44, VS4-6

MOHD ROSLI MB, UP3-56

MOIN T, MP33-4

MOKOOL L, MP32-20

MOLDOVEANU C, MP2-7, MP3-4, UP1-5

MOLINA W, MP27-8

MOLINA WR, MP5-19

MOLISSO G, UP5-26, VS12-8

MOLTZAHN F, MP19-2

MONAHAN K, MP15-9, MP15-10, UP2-28, UP2-34, UP5-52

MONCLESI S, MP23-20

MONDA S, MP8-2, MP8-3

MONGA M, MP2-12, MP4-11, MP26-20, MP27-15, MP28-17, MP28-22, UP1-1

MONTANARI E, MP5-7, MP22-1, MP30-6, MP31-3, UP1-44, UP1-45, UP4-8, VS15-3

MONTERO FABUENA R, VS2-2, VS15-9

MONTORSI F, MP16-17, MP18-21, MP23-6

MOON H, MP17-17, MP26-6

MOON K, UP1-42, UP4-42

MOORE C, MP4-12

MOORE E, MP24-12

MOR Y, UP5-31

MORABITO F, VS14-7

MORADI G, MP9-9

MORADZADEH A, UP4-49

MORALES MONTOR J, UP4-11, VS6-11, VS18-8

MORAN NK, UP1-1

MOREIRA P, MP28-4

MORELLI M, MP31-3

MORENO PALACIOS J, MP24-3, UP3-49

MOREY M, MP24-12

MORGANSTERN B, MP7-20

MORI H, MP33-13

MORI N, MP15-18

MORINAGA S, MP29-13

MORITA K, MP19-6, UP3-36

MORIZANE S, MP15-11, MP15-19, UP2-36, UP2-37, VS17-11

MOROZOV AO, VS7-10, VS12-4, VS15-10

MORRA I, MP14-10, MP15-4

MORRISON I, MP4-10

MORSI H, MP21-1

MOSAYYEBI A, UP1-26

MOSBAH A, VS4-1

MOSCHONAS D, MP18-7, MP30-5, UP2-18, UP2-53

MOSEY R, MP1-15

MOSQUERA L, VS1-9

MOSQUERA M, MP20-17, UP2-56
MOSTAFID H, MP30-5, UP2-18

MOTA P, MP8-4, UP4-21

MOTAMEDINIA P, MP13-12, MP27-16

MOTERIA S, MP12-18, MP26-7

MOTTA G, MP11-22, MP27-17, MP30-6, UP3-16, UP3-28, UP3-58

MOTTLEY C, MP22-10

MOUFID K, VS8-11

MOUSAVI-BAHAR S, UP4-7

MOUTON M, MP11-14, UP3-59

MUELLER T, UP3-15

MÜFÜTOĞLU M, MP1-8

MUHAMMED K, MP24-4

MÜHLSTÄDT S, UP5-17

MUKAI K, UP2-24

MULTESCU R, MP24-1, MP29-1, UP1-5, UP4-39, UP4-45, UP5-51

MUNVER R, MP18-6, MP20-15, MP22-6, MP23-3, MP25-20, UP2-7, UP3-24, UP3-25, UP5-30

MURAI S, MP19-6, MP19-11, UP3-36, UP4-59, UP5-28

MURAKAMI M, MP15-20

MURAMATSU H, MP29-13

MURATA D, MP30-15

MURATA S, MP30-3

MURPHY C, MP31-16

MURRAY K, MP30-19

MURUGAPPAN S, MP24-12

MUSSO G, MP5-2

MUSTAPHA L, MP9-8

MUTO G, VS14-6

MUTO S, MP28-11

MUTTIN F, MP16-17

MYAJIMA N, UP5-28

MYNDERSE L, UP3-19

$\mathbf{N}$

NA J, VS7-12, VS15-13

NA S, MP15-9, UP2-28

NABEEH H, MP11-1

NABI G, MP19-9

NACHIMUTHU S, VS6-4, VS6-6, VS14-3

NADLER RB, MP20-4, MP25-17

NAGAHARA A, MP17-16, UP1-57

NAGAI A, VS17-1

NAGATA M, MP28-11

NAGLE A, MP22-7, UP5-42

NAIDOO T, MP22-4

NAIN A, MP22-10

NAIR R, MP30-8, UP5-47

NAIR S, MP1-1

NAITOH Y, MP16-16

NAKADA SY, MP8-19, MP10-13, MP10-14, MP28-3, MP28-20

NAKAGAWA M, MP17-11, MP21-21

NAKAI Y, UP2-38

NAKAJIMA H, UP2-43

NAKAJIMA S, MP25-5

NAKAMURA K, MP29-13

NAKANE K, VS13-6

NAKANO M, UP6-53, VS13-6

NAKANO Y, MP15-8, MP16-14, MP20-20

NAKAYAMA M, UP2-38

NAKAZAWA S, MP17-16

NALAGATLA S, MP7-18, MP32-20, UP4-4 
NAM J, MP10-10, MP22-8

NAM W, UP3-47

NAMDARIAN B, MP17-2, MP22-5, MP25-10

NANE I, MP8-21, MP17-22, MP24-9, MP32-16, UP4-3, UP6-29

NAPODANO G, UP5-26, VS12-8

NARA T, MP25-5, UP2-46, VS8-12

NARAHARI K, MP28-12

NARANG G, MP10-12

NARITA M, MP14-20, VS12-12

NARITA S, MP25-5, UP2-46, VS8-12

NARUKAWA T, MP16-16

NARYSHKIN S, MP30-20

NASSERI R, MP18-21, MP23-1, MP23-6, UP5-4

NASTOS K, MP27-3

NASU Y, MP9-18, MP15-16, MP25-9, MP25-11, MP30-16, UP2-22, UP2-39, UP6-55

NASUTION PD, MP21-20

NATSUYAMA T, MP20-8

NAVARATNAM A, UP3-11, UP5-27, VS13-4

NAVETTA AF, MP22-21

NAYAK B, MP1-13

NAYYAR R, MP1-14

NAZZANI S, MP11-22, MP27-17, MP30-6, UP3-16, UP3-28, UP3-58

NEAL N, MP24-17

NEEMAN A, UP2-5

NEGRO C, UP3-12, VS3-13, VS14-7

NELSON CP, MP26-5

NESTEROV D, MP31-21

NETSCH C, MP2-13, MP3-1, MP3-5, MP3-17, MP6-17, MP11-5, MP11-9, UP3-13, UP6-46

NEUMANN K, MP17-14

NEVO A, MP8-5, MP31-5, UP6-11

NEW F, MP29-4

NEWMAN MW, MP28-10

NG A, UP5-50, UP6-43

NG C, MP9-15, MP30-14, UP4-16

NG M, UP3-31

NG T, UP4-16

NGO X, MP17-13, VS13-2

NGUYEN A, MP2-12

NGUYEN B, MP8-8

NGUYEN D, MP17-13, VS13-2

NGUYEN G, UP1-55

NGUYEN K, VS18-13

NGUYEN M, UP2-51

NGUYEN T, MP17-13, VS13-2

NI RAGHALLAIGH H, UP6-17

NICULESCU R, MP15-4, UP2-33

NIJENHUIS M, UP6-58

NINI A, MP16-17

NIR T, MP29-10

NISHIKAWA G, MP29-13

NISHIKAWA R, MP15-19, UP2-36, UP2-37, VS17-11

NISHIKAWA Y, VS17-11

NISHIKIMI T, MP23-10

NISHIMURA K, UP2-38

NISHIMURA S, MP25-9, MP25-11, MP30-16, UP2-22

NISHIOKA S, MP20-20

NIU Y, MP23-8

NOAH K, MP11-15

NODA T, UP2-43

NOËL JP, VS9-5
NOELL B, UP1-39

NOENNIG B, MP4-14

NOGUCHI G, UP5-35

NOGUEIRA L, MP27-8

NOGUEIRA M, MP22-3, MP25-22

NOMA Y, MP28-11

NOMURA M, MP33-13

NOMURA N, MP30-15

NONOMURA N, MP17-16, UP1-57

NOTT L, MP10-1

NOTTINGHAM C, MP6-14, VS3-3

NOUR H, UP1-9

NOURALIZADEH A, MP32-1

NOURELDIN YA, MP11-15, UP3-39, UP4-60

NOYOLA GUADARRAMA A, MP2-16

NSEYO U, VS8-8

NTASIOTIS P, MP3-9, MP21-2, UP6-30

NUMAKURA K, MP25-5, UP2-46, VS8-12

NURCHOLIQ S, MP26-13

O

O DE, MP17-18, MP17-21

O'CONNELL H, UP3-6

O'LEARY ML, MP5-21, MP19-13, MP19-14, MP22-14, MP27-20, UP1-19

OADES G, UP5-50

OBAID K, MP33-18

OBAID MA, MP22-9

ÖBEK C, MP1-8, MP6-11, MP16-2, MP18-1, MP20-10, UP1-48, VS9-7

OBIDNYAK V, MP5-22, MP10-7

OBI-NJOKU O, MP21-5, UP3-14

OCCHINO J, VS6-12

ODA H, UP2-35

ODA T, MP20-20

ODEH RI, MP17-18, MP17-21

OEDA T, MP9-18

OGAN K, MP8-12

OGATA A, UP3-1

OGAWA S, MP15-8, MP20-20

OGAWA T, UP6-53

OGDEN CW, VS9-5

OH C, VS11-12

OH J, MP6-13, MP16-21

OH K, UP4-52

OH S, UP1-7

OH T, MP21-6

OHARA S, MP30-15

OHIRA S, VS17-1

OHTA S, MP20-8

OHTAKE S, MP16-1, MP19-10, MP19-12

OJEA CALVO A, VS2-2, VS15-9

OJHA S, MP20-12, MP30-17, VS7-9, VS17-6

OKA T, UP2-38

OKADA K, MP15-18, UP2-19

OKAFOR H, VS15-15

OKEKE Z, MP7-20

OKHUNOV Z, MP12-22, MP21-13, MP27-20, MP31-8, UP1-27, UP5-29

OKINAKA Y, VS12-12

OKUDA Y, UP2-38

OKUSA T, MP15-18, UP2-19

OLIVEIRA C, MP8-4, UP4-21

OLIVER R, MP29-4 
OLSBURGH J, MP22-7

OLSEN M, MP8-2, MP8-3

OLSZYK R, MP13-12

OLVERA-POSADA D, UP6-14

OLWENY EO, MP8-6, UP4-30, UP6-19

OMAR H, MP21-14

OMAR M, MP29-21

ÖNAL B, UP2-20, UP4-22, UP6-48

ONER S, UP5-46, VS6-2, VS8-4, VS13-1, VS13-7, VS14-11

ONOL F, MP15-21, MP15-22, MP20-16, VS5-4,

VS9-1, VS9-11

ONYEZE CI, UP1-2

OPPEZZI F, MP32-2, MP32-10

ORDAZ JURADO D, VS2-8, VS15-14

ORDON M, MP7-19, VS16-1, VS16-2

ORDONEZ M, MP21-19

ORLOV I, UP3-41

ORTEGA GONZALEZ M, UP4-11, VS6-11, VS18-8

OSANN K, MP31-8

OSAWA T, MP19-6, MP19-11, UP3-36, UP4-59, UP5-28

OSMAN BA, MP1-15

OSORIO-GARCÍA MA, UP6-14

OSTHER PJ, MP2-10, MP2-11, MP24-17

OSTROWSKI A, VS5-9

OSUGA K, UP1-57

OTSUKI H, MP9-18

OTTO B, MP10-16, MP12-19

OUSSOREN H, MP21-16

OWUSU R, VS8-9

OWYONG M, MP5-16, MP19-13, MP19-14, UP1-52

OYAMA M, UP2-6, UP2-15

OZAKI K, MP33-13

OZAWA K, VS13-6

OZCAN F, MP4-4, MP4-5, MP8-21, MP17-22, MP24-9,

MP32-16, UP1-36, UP4-3, UP6-29, VS7-7

OZCAN S, MP26-10

ÖZDEN E, MP33-18

OZDEN E, UP5-46, VS6-2, VS8-4, VS13-1, VS13-7, VS14-11

OZEN M, VS13-1

OZERCAN A, MP24-4, MP29-20

OZGUR G, MP26-12

OZIMEK T, MP10-19, MP29-14

OZKAN O, UP4-31

ÖZKENT S, VS5-1

ÖZLÜLERDEN Y, MP32-13

ÖZMAN O, UP2-20, UP4-22, UP6-48

OZMEZ A, UP4-3

ÖZTÜRK A, VS1-7, VS5-1

$\mathbf{P}$

PARK B, MP23-5

PR S, UP6-10

PACE K, MP7-19

PACELLA C, MP6-18

PACHECO GAHBLER C, MP7-21, UP4-11, VS6-11, VS18-8

PADUA HM, MP26-5

PAGADOR J, MP19-21

PAGANELLI G, MP6-18

PAI A, UP6-17

PAIN J, MP20-2

PAIS V, MP8-19, MP28-3, MP28-20, MP28-22

PAKMANESH H, UP4-23

PAL P, MP8-16, MP13-1, MP27-7, MP31-10, UP1-54, VS12-3
PALANEER S, MP19-1, MP21-3, UP4-58

PALAYAPALAYAM GANAPATHI H, MP15-21, MP15-22, MP20-16, VS5-4, VS9-1, VS9-11

PALERMO G, MP1-4, UP1-8

PALKA J, MP8-7

PALMER E, MP2-15

PALMISANO F, MP5-7, MP22-1, MP31-3, UP1-44, UP1-45, UP4-8, VS15-3

PALOU J, MP21-11, MP21-12, VS1-9, VS15-8

PAN S, MP4-12

PANACKAL A, UP5-45

PANAGIOTA T, MP15-19, UP2-37

PANDEY S, MP14-22, MP17-6

PANGILINAN J, MP1-11

PANWAR G, MP26-7, UP6-50

PAPA N, MP29-16

PAPAGIANNOPOULOS D, MP8-8, MP22-16

PAPALIA R, VS14-6

PAPATSORIS A, MP27-3, UP6-4

PARADIS A, MP16-12, MP25-7

PARADISO M, VS3-13

PARDO A, MP6-3

PAREEK G, MP8-9, MP32-6

PAREKH A, MP15-3

PAREKH K, MP17-21

PARI M, UP4-5

PARIKH S, MP12-6

PARINI M, MP16-20, VS14-13

PARK B, UP2-47, UP6-44

PARK D, MP18-12

PARK E, MP18-20, VS2-10, VS11-12

PARK I, MP6-13

PARK J, MP13-15, MP29-15

PARK K, UP4-52

PARK S, MP1-17, MP10-10, MP22-8, MP25-6, MP33-7, UP1-42, VS2-10, VS5-13, VS11-12

PARK W, MP18-20

PARKER A, MP18-11

PARKHOMENKO E, MP12-22, MP19-13, MP19-14, MP22-14, MP32-5, UP1-52, UP5-29

PARMAR KM, MP10-8, VS2-1, VS8-7, VS16-12

PARRA-DAVILA E, MP20-16

PASQUIÉ M, UP3-23

PASSOS P, VS11-1

PATEL A, MP1-5, MP30-11, VS7-3

PATEL B, MP24-10, MP33-15

PATEL C, MP21-5

PATEL D, UP4-49

PATEL H, MP19-3

PATEL K, MP27-11, UP3-46

PATEL N, MP11-16, MP12-6, VS6-8, VS6-13, VS7-5, VS18-6

PATEL R, VS18-4

PATEL RM, MP2-14, MP5-16, MP5-21, MP12-22, MP19-13, MP19-14, MP21-13, MP22-14, MP27-20, MP31-8, UP1-19, UP1-27, UP1-52, UP5-29

PATEL S, UP5-4, VS6-1, VS8-8, VS12-5

PATEL V, MP15-21, MP15-22, MP20-16, VS5-4, VS9-1, VS9-11

PATELLI G, MP6-18

PATERSON R, MP2-17, MP9-16

PATHAK A, MP15-3

PATHAK R, MP23-2, VS5-5, VS9-10

PATHAK S, VS12-3 
PATIL D, MP8-12

PATIL K, MP30-5, UP2-18, UP2-53

PATIL SR, MP20-12, MP30-17, VS7-9, VS17-6

PATKI P, MP27-7

PATTERSON J, MP19-15

PATTERSON L, MP20-14

PATWARDHAN S, UP6-28

PAULUCCI D, MP16-5, MP16-18, MP18-3, UP2-50, UP5-1

PAVAN N, MP18-21

PAVLAKIS P, MP18-7, MP30-5, UP2-18, UP2-53

PAVLINEC J, MP4-14

PEACOCK J, MP4-18

PEARLE MS, MP8-17, MP8-18, MP27-13, UP6-22

PEARLMAN AM, MP6-19

PECORARO A, MP14-10, UP2-33, UP5-38

PEDERSEN MR, MP2-10, MP2-11

PEDNEKAR A, VS7-6, VS17-8, VS18-2

PEDROSA I, UP1-34, UP1-38

PEIXOTO GA, UP1-1

PELGER R, UP6-58

PELIN D, VS13-9, VS14-4

PENA LA-GRAVE GE, VS18-1

PENA-LA GRAVE GE, VS18-1

PENEV B, UP3-32

PENG Y, MP5-10, UP3-35, UP6-42

PENHALEUX N, MP27-4

PENNISTON KL, MP2-9, MP2-15, MP8-18, MP8-19,

MP10-11, MP10-13, MP10-14, MP28-3,

MP28-20, UP6-23

PEREIRA J, MP8-4, MP8-9, MP32-6

PEREIRA-SAMPAIO M, MP1-2, MP1-3

PERERA M, MP11-17, MP29-16, UP1-13

PERETTI D, MP14-10, UP2-33

PÉREZ SCHOCH M, VS2-2, VS15-9

PEREZ-ARDAVIN J, VS2-8, VS15-14

PEREZ-FENTES D, MP24-17, MP27-12

PÉREZ-LANZAC A, VS11-5

PERROUIN-VERBE M, MP12-21

PERRY KT, MP20-4, MP25-17

PERRY M, MP30-5, UP2-18, UP2-53

PERSAD R, MP18-8

PESQUE R, MP27-5

PETERS B, UP1-50

PETERS C, MP27-18, MP29-12

PETERS J, UP5-40

PETKOVA K, MP26-11, MP32-14

PETRIDES N, MP4-10

PETROV S, MP5-22, MP10-7

PEYRONNET B, UP3-23

PHAM B, MP32-7, UP4-54, UP4-55

PHAN Y, MP27-10, UP3-56

PHILIP J, MP7-8, MP13-13, MP13-14, UP1-17

PHILLIPS J, MP11-16, MP12-6

PHUNG MC, MP1-11, MP3-6, MP4-9, UP2-51, UP3-22

PIAGGIO G, MP1-7

PIANA A, MP14-10, UP2-33, UP5-38

PIAZZINI A, MP6-18

PICHARDO M, MP22-12

PICKERSGILL NA, MP4-7, MP21-7, MP23-12

PICKHARDT M, VS10-9

PICKLES T, MP13-13, MP13-14

PICONI S, MP22-1

PICOZZI S, UP3-28, UP3-58

PIEN L, MP9-7
PIERAS E, VS8-10

PIESCHE S, MP6-22

PIETROPAOLO A, MP6-8, MP7-14, MP24-20,

MP27-19, MP32-8

PILISIU A, MP6-18

PINDORIA N, MP11-18

PIÑEIRUA-MENENDEZ A, MP12-1

PING H, MP23-8

PINI G, MP19-15

PIÑON SOLIS E, MP2-16

PIOTROWSKI J, VS9-4

PIRAMIDE F, MP15-4, UP2-33

PISAREV A, MP5-22, MP10-7

PIZA P, VS8-10

PIZZI G, VS17-9

PLASENCIA G, UP5-33

PLATA M, MP3-7, UP1-6

PLEASS H, VS13-5

PLOUMIS K, UP3-45

PNG K, MP13-22, MP16-10, MP19-22, UP5-43, VS18-3

PODE D, UP1-46

POGGIO E, MP4-11

POGGIO M, MP15-4

POLGREEN P, MP24-8

POLOTTI CF, VS18-4

POMANN G, MP9-11

POMPEO V, MP1-7

PONZONI A, MP5-2

POPERT R, MP11-18, MP22-5

POPIOLEK M, MP10-7, MP29-18

POPOV S, UP3-41

PORPIGLIA F, MP6-1, MP14-10, MP15-4, MP16-13,

UP2-33, UP5-38

PORTER J, MP16-5, MP16-13, MP23-6, MP25-16,

UP2-50, UP5-1

PORTNOY O, UP5-31

POSIELSKI N, MP10-14

POTHIYEDATH H, VS3-9, VS5-8

POTRETZKE AM, MP16-12, MP18-9, MP25-7, VS15-2

POTRETZKE TA, MP18-9

POURNARAS C, MP9-21

PRADERE B, UP3-23

PRAHARA Y, MP26-13, UP4-25

PRASAD S, MP1-1

PRASAD V, VS11-2, VS11-3, VS11-4, VS13-3, VS14-8

PRASHANT Y, VS11-2, VS11-3, VS11-4, VS13-3, VS14-8

PRATTLEY S, MP29-4, UP1-28, UP5-58, UP6-38

PREISSER F, MP30-6

PREMINGER GM, MP10-20, MP27-18, MP28-1, MP29-12, UP1-55, UP6-1

PRESS B, MP18-6, MP23-3, MP25-20, UP2-7, UP5-30

PREZA-FERNANDES J, VS11-1

PRICE ET, MP3-6, UP3-22

PRÍNCIPE P, UP5-53, VS8-6

PRODIGALIDAD JT, MP17-3

PROIETTI S, MP24-17, MP24-20, UP4-24, VS1-12

PROSKURA A, MP1-6, MP30-21, VS4-7

PROSPERI M, UP5-41

PROWSE O, UP3-7, UP3-8, VS3-2

PRYTUŁA A, MP9-7

PRZUDZIK M, VS6-14

PU Y, MP16-19

PURNELL S, MP24-22, MP29-5, MP32-5, UP6-6, UP6-20

PUROHIT R, VS18-9 
Q

QADRI S, MP26-16, MP26-17

QIAO Y, MP13-22

QIN C, MP1-12

QU G, MP1-21

QUACH D, MP17-13, VS13-2

QUADRINI F, UP5-14

QUIRKE K, MP19-15, MP19-18, UP5-59

$\mathbf{R}$

RABAH B, MP9-8

RABANI S, UP6-21

RABINOVICH I, UP1-46

RACIOPPI M, MP1-4, UP1-8

RADFAR M, MP32-1

RADTKE A, VS9-4

RAFFIN E, MP8-19

RAGAB M, MP33-14

RAGAM R, MP18-6, MP23-3, MP25-20, UP2-7, UP5-30

RAGHAVENDRAN M, MP27-11, UP3-46, UP3-57

RAGNI F, MP15-4, UP2-33

RAGONESE M, MP1-4, UP1-8

RAGOORI D, VS8-5

RAI A, MP19-16, MP19-17

RAI B, MP6-8

RAI S, MP11-10, UP5-39

RAIS R, MP25-7

RAISON N, MP19-2, MP19-6, UP3-36

RAJ M, UP3-6

RAJA A, MP13-13, MP13-14

RAJAK Y, MP29-3

RAJANGAM A, MP25-10

RAJJAYABUN P, MP13-18

RAJPUT M, UP1-37

RAMACHANDRA MN, MP8-10

RAMACHANDRAN N, MP7-15

RAMADAN A, MP33-14

RAMANI S, MP27-7, VS7-2, VS7-6, VS7-8, VS9-2, VS10-4, VS12-7, VS15-7, VS17-8, VS18-2

RAMASWAMI K, VS3-9, VS5-8

RAMON J, UP4-51, UP5-31

RANASINGHE S, MP11-17

RANFORD D, MP10-15

RAPOPORT L, MP1-6, MP2-13, MP3-1, MP3-17, MP6-20, MP11-12, MP11-21, MP14-17, MP30-21, UP6-46, VS1-3, VS3-1, VS3-5, VS3-10, VS4-2, VS4-7, VS7-10, VS9-12, VS12-10, VS15-10, VS17-10

RAPPAPORT Y, UP1-31

RASHID S, MP13-18

RASSWEILER J, MP5-3, MP19-2

RASTINEHAD A, MP1-1, MP4-3, MP23-13, UP1-35

RASYID N, MP12-7, MP26-1

RATAN H, UP4-50

RATHOD J, MP10-15

RATNER B, MP2-14

RATTI D, MP11-22, MP30-6, UP3-16

RAUDABAUGH J, UP1-55

RAWAL S, MP17-1

RAZVI H, MP10-1, UP1-16

REALFONSO T, UP5-26, VS12-8

RECALDE LARA C, UP5-54

REDDY M, MP23-1, UP5-4, VS8-8, VS8-9

REDMOND E, MP31-16

REDSHAW JD, MP25-1
REGAN D, MP22-14

REGIS F, MP21-11

REGUSCI S, MP6-18

REISZ PA, MP11-2

RELIFORD-TITUS S, MP10-18, MP28-3, UP1-53

REMER E, MP4-11

RENGIFO-ABBAD D, MP25-3, VS17-5

RENTERIA J, MP16-11

REYNOLDS LF, MP7-19, VS16-1, VS16-2

RHA K, MP18-14, MP18-21, MP23-6, MP32-12,

UP5-9, VS7-12

RHEW H, MP25-6

RICHSTONE L, VS18-14

RIEKER P, MP5-3

RIERA L, MP7-11

RIMINGTON PD, MP30-1

RINCÓN-PEDRERO R, MP2-8

RISAN M, MP26-13

RITCHIE C, MP15-14

RITTENBERG D, VS16-9

RIVERA ME, MP2-1, MP4-21, MP31-1, MP33-19, UP1-14, UP3-19, VS3-4

RIZZO G, MP6-4, MP23-20, MP30-12

RIZZO M, MP1-7

ROBERTS M, UP1-13

ROBERTS WW, MP24-6, MP24-11, MP27-1, UP4-32

ROBLES J, MP6-19, MP11-2, MP28-19

ROCCO B, VS9-1

ROCCO N, MP17-5

ROCHA B, MP13-9, MP13-10

ROCHESTER M, MP11-3

RODGER F, UP5-50, UP6-43

RODRIGUEZ LAY R, MP22-12, MP23-15, UP1-59

RODRIGUEZ-MONSALVE HERRERO M, MP2-5, MP10-2, MP18-10, MP19-4, MP25-4, UP1-21, UP5-3, UP5-16, VS1-10, VS2-5, VS16-14, VS17-5

RODRÍGUEZ-REINA G, UP5-3

RODRIGUEZ-RUBIO F, VS11-5

RODRIGUEZ-SOCARRAS M, MP24-20, UP4-24, VS1-12

ROEHRBORN CG, MP6-5, MP11-7, MP11-12

ROGERS A, MP7-5, MP8-11

ROGERS C, VS7-3

ROGERS T, MP11-13, MP15-21, MP15-22, MP20-16, VS5-4, VS9-1, VS9-11

ROKBA MA, MP11-4

ROLO F, MP28-16

RONDÓN M, UP1-6

ROODHOUSE A, MP30-5

ROOF S, MP15-21, VS5-4

ROSA R, MP16-11

ROSE E, MP2-12, MP28-17

ROSEN G, MP28-2

ROSLAN M, VS6-14

ROSOFF JS, MP30-2

ROSSO M, MP24-20

ROSTAMINEJAD N, VS4-4

ROTH G, MP25-15

ROTHBERG M, UP5-1

ROUMEGUÈRE T, MP24-18

ROUSE A, MP4-9

ROUSE L, MP29-6

ROUSE PH, MP30-1

ROUSSELLE S, MP3-12

ROUTH JC, MP9-11, MP14-19, MP28-1 
ROZET F, MP23-14

RUANO MAYO A, MP3-11

RUCHI R, UP6-33

RUCKLE HC, UP2-31

RUDD I, MP20-2

RUGGERA L, MP5-2

RUİZ HERNÂNDEZ J, MP21-8

RUKSTALIS D, MP11-7

RULE AD, MP2-1, UP1-14

RUOSS J, MP4-14

RUSSEL K, UP6-45

RUSSELL B, VS9-5

RUSSELL D, MP33-11

RUSSO F, UP5-38

RYAN S, MP23-1, MP23-6, UP5-4, VS8-8, VS8-9

RYAN K, VS18-1

RYU K, MP21-18, UP6-44

\section{$\mathbf{S}$}

S.M.L. P, MP3-22

SAAD IR, MP30-4

SAAD S, MP17-2, MP30-8, UP5-47

SABAA M, MP33-14

SABATINI I, MP31-3, UP1-44, UP4-8, VS15-3

SABBAGH S, UP3-30

SABELA L, VS15-9

SABER-KHALAF M, MP3-14, MP9-6

SABHARWAL T, MP11-18

SABNIS R, MP7-6, MP18-2, MP31-22, UP3-43,

UP6-52, VS12-1

SACCO D, VS5-12

SACCO E, MP1-4, UP1-8

SADAHIRA T, MP9-18, MP15-16, MP25-9, MP25-11, UP2-22, UP2-39, UP6-55

SAEED IA, MP2-15

SÁENZ-MEDINA J, MP16-20, VS2-5, VS17-5

SAFAEE ARDEKANI G, MP9-16

SAFIULLAH S, MP19-13, MP19-14

SAGALOVICH D, MP16-6, MP16-15, MP18-4, MP23-18, MP25-13, MP30-10, UP2-1, UP2-12, UP5-2, VS8-10

SAĞLAM R, MP6-2

SAĞLICAN Y, MP1-8

SAGY I, UP1-20

SAHAN A, MP26-19

ŞAHIN A, UP1-48

SAHIN B, MP22-22

SAHIN S, MP20-7, MP20-17, UP2-56, VS9-6

SAHU M, UP2-23

SAID M, MP8-12, VS16-11

SAIDANI M, UP4-38

SAIKA T, MP9-18, UP2-43

SAINI S, MP1-14

SAITO M, MP25-5, UP2-46, VS8-12

SAITTA G, MP24-20, UP4-24, VS1-12

SAKAI A, MP22-3, MP25-22

SAKAI H, MP15-7

SAKAMOTO N, UP3-1

SAKI ZI, MP3-9, MP21-2, UP6-30

SALAKO AA, MP3-2, UP1-2

SALCEDO J, MP23-16, MP23-17

SALEEM M, VS3-9, VS5-8

SALEEMI A, MP17-19, UP3-14

SALEH G, UP4-9

SALIB M, MP4-18
SALKINI M, MP8-13, MP14-16, MP23-4

SALONIA A, MP22-1, UP1-44, UP1-45, UP4-8

SALTIROV I, MP26-11, MP32-14

SALVIZ E, MP17-22

SAM E, VS9-6

SAMAHA I, MP26-9

SAMATEH A, MP30-1

SAMI S, UP2-54

SAMPAIO FJ, MP1-2, MP1-3, MP29-7

SAMPOGNA G, MP5-7, MP22-1, MP31-3, UP1-44, UP1-45, UP4-8, VS15-3

SAMSON P, MP7-20

SAMY K, MP9-8

SANCHEZ AQUINO U, MP7-21

SÁNCHEZ GARCÍA J, VS2-2

SANCHEZ MACÍAS J, MP6-3

SÁNCHEZ MARGALLO F, MP19-21, MP27-12

SÁNCHEZ RAMOS J, VS2-2, VS15-9

SÁNCHEZ F, VS1-9

SANCHEZ H, UP4-17, UP4-18

SANCHEZ-GONZALEZ J, VS15-14

SÁNCHEZ-GONZÁLEZ J, VS2-8

SÁNCHEZ-GUTIÉRREZ FJ, MP11-19, UP4-17, UP4-18

SANCHEZ-SALAS R, MP23-14, VS8-3

SANCI A, MP12-2

SANDBÆK G, MP16-3

SANDERS A, MP10-17

SANGHVI N, MP22-11, UP5-15, UP5-32

SANGUEDOLCE F, MP21-11

SANLI O, MP4-4, MP4-5, MP8-21, MP17-22, UP1-36

SANO M, UP2-30

SANSEVERINO R, UP5-26, VS12-8

SANTONI N, UP5-50, UP6-43

SARICA K, MP19-15, MP26-10, UP5-59

SARIDOGAN E, MP14-15

SARIKAYA S, UP3-35

SATHIANATHEN N, MP10-3

SATKUNAS HN, UP1-1

SATO F, UP2-49

SATOH S, UP2-46, VS8-12

SAULAT S, MP26-16, MP26-17

SAVRAN KARADENIZ M, MP17-22

SAWADA Y, UP2-43

SAWANAPORN R, MP8-14

SAYYID R, MP20-3

SCALES CD, MP9-11, MP10-20, MP27-18, MP28-1, MP33-4, UP1-55, UP6-1

SCALES J, VS6-7

SCARBOROUGH P, MP17-5

SCHEITLIN W, MP5-3

SCHERMERHORN S, MP17-5

SCHIPS L, MP16-13

SCHLAGER D, MP22-9

SCHLEICHER I, UP3-45

SCHNEIDER MH, MP10-19, MP29-14

SCHOEB DS, MP22-9

SCHÖNTHALER M, MP22-9

SCHOUT B, UP6-58

SCHULMAN A, MP11-16, MP12-6

SCHULMAN C, MP6-1

SCHULSTER M, MP17-12

SCHUMACHER A, UP5-56

SCHUMANN A, UP5-17

SCHWARTZ J, MP4-3 
SCHWEN Z, MP5-13

SCOFFONE CM, UP3-3, UP3-4, VS3-6

SCOTLAND KB, MP2-17, MP9-16, UP1-29

SCOVELL J, MP19-16, MP19-17

SEBAEY A, MP11-15

SEE K, MP15-12, MP22-19

SEIFMAN BD, MP28-9

SEITZ C, MP19-15, MP24-17

SEKERCI C, MP26-19, UP4-26, UP4-31, UP5-49, VS6-9

SELIM MA, MP11-4, UP4-9

SEMINS MJ, MP4-19, MP22-15, MP22-17, MP33-6, UP3-44, UP6-11, VS4-6

SEN A, MP2-18, UP1-30

SENER G, MP2-18, UP1-30

SENER T, MP2-18, MP19-4, MP22-22, MP26-12, MP26-19, UP1-22, UP1-30, UP4-26, UP4-31, UP5-49

SENTINELLI S, UP1-56

SENTURK CIFTCI H, MP17-22, UP4-3

SENTURK A, UP6-12

SEO D, UP3-53

SEO J, MP3-19, MP15-13, MP18-13, MP20-11, MP25-19, UP5-10

SEO S, MP18-12, UP5-21

SEO W, MP25-6

SEOW C, UP1-29

SERNI S, MP20-17, UP2-56

SERRANO A, MP27-12

SERRANO D, MP15-17

SERVICE A, MP25-1

SETH A, MP1-13

SEVERGINA L, MP1-6

SEWELL J, UP3-6

SEYAM RM, MP25-14

SEYED H, UP3-17

SFAKIANOS J, MP4-3, MP19-2, MP23-13, UP1-35, UP5-1 SHAH A, MP8-5

SHAH M, MP7-3, MP7-4

SHAH N, UP6-35

SHAH NL, UP5-5

SHAH O, MP9-13, MP28-2, MP28-22, MP31-17

SHAH P, MP12-18, MP26-7, UP3-27, UP6-50

SHAH Q, MP18-3, UP5-6

SHAHAIT M, MP8-5, MP15-9, MP15-10, MP22-10, MP22-15, MP23-7, UP2-28, UP2-34, UP5-52, UP6-11

SHAHBAZ S, MP13-21

SHAHID A, MP8-16, UP1-54, VS12-3

SHAHROUR W, UP3-7, UP3-8, VS3-2

SHAKHSE SALIM N, MP32-1

SHAKIBA B, VS4-4

SHAMAN J, MP28-2

SHAMIEH B, MP9-1

SHANMUGATHAS N, MP4-18

SHAO P, MP1-12

SHARIFI AGHDAS F, MP32-1

SHARMA A, MP1-14

SHARMA AP, MP10-8, VS2-1

SHARMA N, MP10-16, MP12-19, UP6-50

SHARMA V, MP33-19, UP3-19

SHATOKHIN M, MP30-20

SHAYEGAN B, MP20-14, UP3-37

SHEIR K, MP9-14, UP3-60

SHEIR KZ, MP9-19

SHEMSHAKI H, MP32-1

SHEN J, UP5-27
SHEN W, MP22-2

SHEN Y, MP20-21

SHENG M, UP4-14

SHEPHERD S, MP33-17, UP3-17

SHERGILL I, MP21-5, UP3-14

SHETTY A, MP4-7, MP4-8

SHI G, UP2-8

SHI X, MP1-10

SHIBUYA T, UP2-48

SHICHINOHE T, UP4-59

SHIDA Y, MP15-7

SHIELDS J, VS2-6

SHILO Y, MP8-1, MP8-15, UP6-13, UP6-37

SHIMBO M, VS9-8

SHIMIZU S, VS17-1

SHIMURA S, MP22-18

SHIN J, UP3-47

SHIN T, UP2-48, UP2-49

SHINDEL A, MP7-7

SHINMEI S, UP2-35

SHINOHARA N, MP19-2, MP19-6, MP19-11, UP3-36, UP4-59, UP5-28

SHIOZAWA S, MP20-8

SHOJI S, UP6-53

SHOKRY A, MP21-1

SHOMA AM, MP9-19, UP4-36

SHOUMAN A, MP21-1

SHPOT E, VS17-10

SHPUNT I, MP8-1, MP8-15, UP6-13, UP6-37

SHRESTHA A, MP29-8

SHUKLA P, MP4-3, MP23-13, UP1-35

SHUM C, MP16-10

SHUMATE A, MP25-15

SHVERO A, UP4-51, UP5-31

SIA J, MP1-19

SIDALSKY S, MP21-17

SIDDIQUE UH, MP8-16, MP31-10, UP1-54, VS12-3

SIDHOM D, MP17-12

SIEGEL YI, UP1-31

SIENA G, MP20-17, UP2-56

SIGNORINI C, MP11-22, MP27-17, UP3-16, UP3-28, UP3-58 SILBERSTEIN J, MP18-15

SILVA MD, MP5-5

SILVA NV, MP12-20, MP26-2, MP32-17, VS1-6

SILVA-RAMOS M, UP5-53

SIM A, MP25-21, VS4-5

SIMMONS W, MP27-18

SIMÕES P, MP28-4

SIMONE G, MP1-7, MP14-12, MP16-13, MP16-17, MP23-6,

MP30-7, UP1-56, UP2-3, VS7-1, VS7-4, VS9-9, VS10-5,

VS16-4, VS17-4, VS17-9

SIMPSON W, MP20-3

SIMSEK A, MP20-7, VS9-6

SIMSEK F, MP26-19, UP4-26, UP4-31, UP5-49, VS6-9

SIMSEKOGLU F, MP13-8

SIMSIR A, VS10-1

SIMSON N, MP4-18

SINGAL A, MP25-17

SINGH A, MP7-6, MP17-1, MP18-2, UP3-43, UP6-52, VS12-1, VS15-15

SINGH D, MP4-12, MP27-16

SINGH P, MP1-13

SINGH S, MP4-6

SINGH SK, UP1-3, VS2-1 
SINGH V, MP14-22, MP17-6

SINGLETON M, MP16-9

SINHA RJ, MP14-22, MP17-6

SIRIPONG N, UP6-11

SIROTA E, VS7-10

SIVALINGAM S, MP5-12, MP28-3, MP28-17, MP28-20, MP29-2

SIVAN B, UP1-20

SJOBERG D, UP1-37

SKARECKY D, MP15-12

SKOLARIKOS A, MP7-14, MP19-2, MP19-15, MP27-3, UP6-4

SKREPETIS K, UP4-44

SLIMANI A, VS8-11

SLONGO J, VS3-12

SLUSARENCO R, VS7-10, VS15-10

SMALL A, MP9-13

SMALL AC, MP12-20

SMALL D, UP5-50

SMITH A, MP7-20

SMITH BG, MP19-18

SMITH D, MP7-2, MP7-12, MP7-22, MP8-11, MP14-7, MP14-15, MP32-15

SMITH JC, UP3-29, UP4-56

SMITS G, UP2-57

SNIR D, MP29-10, MP32-18

SNURNITSYNA O, MP14-17, VS12-10

SOANS J, UP3-30

SOARES RO, MP20-4, MP25-17

SOBEL DW, MP29-9

SOBOL I, UP1-14

SOBRINHO UG, MP29-7

SODA G, MP23-20

SOERENSEN FB, MP2-10, MP2-11

SOEROHARJO I, MP26-13, UP4-25

SOEROTO AA, MP26-1

SOFER M, MP26-20, MP29-10, MP32-18

SOFIMAJIDPOUR H, MP9-9

SOLEIMANI B, UP4-10

SOLIMAN M, MP33-14

SOLOMATNIKOV I, MP23-19

SOLTAN M, MP11-1

SOMANI BK, MP6-8, MP7-14, MP8-10, MP9-2, MP9-3,

MP13-13, MP13-14, MP19-4, MP19-15, MP24-9, MP24-20, MP27-19, MP28-21, MP31-9, MP31-14, MP32-8, MP33-1, UP1-22, UP1-26, UP5-58, UP6-34, UP6-38, UP6-39

SOMUNCU B, MP1-8

SON H, MP13-15, MP29-15

SONAWANE PL, UP6-52

SONG B, MP16-21, MP30-22, UP3-38

SONG L, MP4-16, MP8-20, MP9-5, MP23-8

SONG S, UP3-47

SONG Y, MP1-17, MP26-14, MP26-15, UP4-57

SÖNMEZ M, VS1-7, VS5-1

SONMEZAY E, MP9-20

SOOD A, MP9-17

SOOMRO A, MP26-16, MP26-17

SOPILKO I, UP6-23

SORENSEN M, MP5-1, MP29-11

SORIA F, MP19-21, MP27-12

SOROKIN I, MP8-17, MP8-18, MP19-8, MP23-11, MP27-13, UP6-22

SOROKIN N, MP1-6, MP30-21, VS1-3, VS4-7

SOTELO R, VS7-1
SOUID M, UP4-38

SOURIAL MW, MP10-5, MP26-18, MP27-14

SPAGNUOLO M, MP1-7

SPERMON R, MP5-17

SPIJKERMAN I, MP21-16

SPILSETH B, MP23-15, UP1-59

SPINELLI M, MP31-3

SPRENKLE PC, UP3-52

SPYROPOULOS K, MP27-3

SRIPRASAD S, MP10-15

SROUGI M, MP13-9, MP13-10

STAMBAKIO H, MP13-5

STANOWSKI M, MP20-2

STEIN R, MP16-6, MP18-4, MP23-18, MP30-10, UP5-2

STEINBERG RL, MP11-12, MP19-8, MP23-11, MP27-13,

UP1-34, UP1-38, UP6-22, VS5-6

STEPHENSON E, MP10-12

STERLING M, MP14-6

STERN K, MP4-11, MP26-20, MP27-15, MP28-20

STERNBERG K, MP29-9

STIFELMAN MD, MP18-6, MP23-3, MP25-20,

UP2-7, UP5-30

STIGLIANO A, UP2-3

STITES J, MP18-6, MP20-15, MP22-6, MP23-3, MP25-20,

UP2-7, UP3-24, UP5-30

STOCKALL ER, MP28-9

STOCKLE M, MP20-17

STOIANOVICI D, MP32-21

STOKES P, MP7-4, MP15-1

STOLLER ML, MP10-18, MP33-2, MP33-3, UP1-53

STONIER T, MP4-18

STREEPER NM, MP8-19, MP10-17, MP13-20, MP28-3,

MP28-20

STREETER EH, MP30-1

STROPE S, MP30-19

STROUP S, MP17-5

STUBINSKI R, UP3-28, UP3-58

STURGEON K, MP17-12, VS16-5

SU B, MP21-9, MP21-10

SU H, MP1-22

SU J, MP4-12, MP27-16

SU L, MP4-14

SUAREZ GM, MP22-11, MP22-12, UP5-15, UP5-32, UP5-33

SUBIELA J, MP21-11, MP21-12, UP2-56

SUBIRÁ RIOS D, MP33-10

SUDHAN H, MP18-16

SÜER E, MP12-2

SUGIE M, MP29-13

SUGIMOTO M, MP25-11, MP30-16

SUGINO Y, MP30-3

SUGIYAMA S, VS17-1

SUKHANOV R, MP30-21, VS4-7, VS12-4

SULEYMAN N, MP4-18, MP17-19

SULTAN N, MP10-1

SULTAN SM, MP11-4

SULTANA A, MP17-18

SUMITOMO M, MP29-13

SUN J, UP2-17

SUN X, MP1-21

SUN Y, MP5-10, UP3-35, UP5-24, UP6-42

SUNG G, MP20-5, UP1-58, UP5-34

SUNG H, MP18-12, UP5-21

SUR RL, MP8-8, MP28-3, MP28-20, MP28-22, MP29-2

SUSHENTSEV N, VS7-10, VS12-4, VS15-10 
SUZUKI I, MP30-3

SVIHRA J, UP6-23

SWINN M, MP30-5, UP2-18

SWORDS K, MP21-15

SYED J, MP4-12, UP3-52

SYMES A, MP13-11

T

TABATA K, MP15-20, MP20-6, MP22-18

TABIB C, VS18-14

TADASHI M, MP19-20

TAGUCHI K, MP7-7

TAILLY TO, MP9-7, MP21-2, MP31-9, UP6-30

TAKAGI T, MP18-21

TAKAHASHI G, UP2-48

TAKAHASHI M, MP33-13

TAKAMOTO A, MP30-16

TAKAMOTO H, MP9-18

TAKAYASU K, MP19-19, MP19-20

TAKAZAWA R, MP29-19

TAKEI K, UP2-49

TAKENAKA A, MP15-11, MP15-19, UP2-36, UP2-37, VS17-11

TAKENAMI T, MP15-20

TAKESHI N, MP16-16

TAKEUCHI S, VS13-6

TAKEZAWA K, UP1-57

TAKHAR M, MP1-1

TALAT Z, UP2-20, UP4-22

TALATI V, MP20-4, MP25-17

TALSO M, MP14-11, MP28-21, VS15-1

TALWAR R, MP13-5

TAM C, MP28-10

TAMADDON K, MP15-3

TAMHANKAR A, MP20-12, MP30-17, VS7-9, VS17-6

TAMIMI A, UP1-31

TAN B, MP8-6, UP6-19

TAN M, MP25-21

TAN MV, MP1-18

TAN T, MP30-8, UP5-43

TAN W, MP21-17, VS18-11

TAN Y, MP1-19, MP25-21, UP2-21

TANEJA S, MP17-19

TANG Y, MP4-13, UP6-24

TANG S, UP6-7

TANIDIR Y, MP22-22, MP26-12, MP26-19, UP3-35, UP4-26, UP4-31, UP5-49, VS6-9

TANIMOTO R, MP15-16, MP25-9, MP25-11, MP30-16, UP2-22, UP2-39, UP6-55

TANWAR HV, UP6-28

TAOULI B, MP1-1, MP4-3, MP18-3, MP23-13, UP1-35, UP5-6

TARATKIN M, MP2-13, MP3-1, MP3-5, MP3-17, MP6-20, MP11-21, UP6-46, VS3-1, VS3-5, VS3-10, VS4-2

TARICIOTTI P, MP6-4, MP23-20, MP30-12

TARIQ A, MP8-16, UP1-54, VS12-3

TAS T, UP6-12

TASCI A, MP20-7, VS9-6

TASIAN GE, MP4-16, MP8-20, MP9-5, MP13-5

TASLEEM A, MP7-15

TATARANO S, MP17-11, MP21-21

TATENUMA T, UP5-35

TATSUGAMI K, VS5-3

TAVARES-DA-SILVA E, MP28-4, MP28-16
TAWFIEK E, MP12-12, MP24-7, MP27-6

TAWFIK A, MP33-14

TAY K, UP2-21

TAZI H, VS2-7, VS8-11

TB Y, VS7-2, VS7-6, VS7-8, VS9-2, VS10-4, VS12-7, VS15-7, VS17-8, VS18-2

TEFIK T, MP8-21, MP17-22, MP24-9, MP28-21, MP32-16, UP4-3, UP6-29

TEISHIMA J, UP2-35

TEJPAUL R, MP10-3, MP21-19

TEMA G, MP23-20

TEMIDO P, MP28-4

TEODOROVICH O, MP30-20

TEOH J, MP9-15, MP30-14

TERACHI T, UP2-49

TERAOKA S, MP15-19, UP2-36, UP2-37, VS17-11

TERLECKI R, MP6-19, MP28-19

TERRAZAS-CERVANTES M, MP2-8

TERRIS M, MP15-14, MP20-3

TERRITO A, MP20-17, MP21-11, MP21-12, UP2-56, UP2-57, VS15-8

TERRY RS, MP4-14, MP10-20, MP27-18, MP28-1, MP29-12, UP1-55, UP6-1, VS10-8

TEWARI AK, MP1-1, MP18-16, MP18-19, UP5-8

THACKER P, MP14-18

THAI J, MP29-5, UP6-20

THAI K, MP17-13, VS13-2

THAI KH, MP24-10, MP33-15

THAI M, MP17-13, VS13-2

THAKER M, UP3-15

THALMANN G, MP19-2

THAVASEELAN S, MP8-9

THIEL DD, MP25-15

THIEL J, MP5-1, MP29-11

THOMAS CS, MP25-15

THOMAS K, UP5-42

THOMAS M, MP4-10

THOMAZ E, MP10-17

THULASIDASAN N, MP11-18

THULASIDASS H, UP5-8

THUM DJ, UP4-49

THURAIRAJA R, MP30-8, UP5-47

TIAN Y, MP2-19, MP9-10

TIE Z, UP3-35

TIMBERLAKE M, MP19-8

TIMONEY A, MP7-8

TIONG H, MP22-13

TIWARI R, UP3-31

TODD AM, MP26-18

TOHI Y, MP30-3

TOKARSKI A, VS6-5

TOKATLI Z, MP6-2

TOLLEFSON MK, MP18-9, VS12-6, VS16-7, VS16-8

TOM W, MP29-3

TONDELLI E, MP14-11, VS15-1

TOOMEY D, UP2-23

TORRECILLA C, MP7-11, MP10-6, UP4-46

TORRECILLA J, MP3-11

TORRES J, MP8-4, UP4-21

TORRICELLI F, MP13-9, MP13-10

TOUSSI A, MP18-9, VS15-2

TRACY C, MP24-8, UP1-37

TRAN N, MP17-13, VS13-2

TRAN P, UP5-57 
TRAN T, MP17-13, VS13-2

TRASSIERRA M, VS2-8, VS15-14

TRAXER OO, MP2-5, MP5-4, MP5-6, MP5-11, MP5-14, MP5-20, MP10-2, MP12-3, MP13-9, MP13-10, MP13-16, MP18-10, MP19-4, MP24-5, MP24-9, MP24-17, MP24-21, MP27-5, UP1-21, UP1-22, UP1-24, UP5-16, VS1-1, VS1-2, VS1-4, VS1-10

TREGUNNO D, MP33-9

TREVISANI F, MP16-17

TRIBUKAIT B, UP5-12

TRIVEDI JR, UP5-39

TRUJILLO CG, MP3-7, UP1-6

TRUONG H, VS6-5

TSAI L, MP22-21, VS12-2

TSAI Y, UP5-7, VS17-2

TSARICHENKO D, VS1-3

TSE C, MP21-13

TSIRKAS K, MP27-3, UP6-4

TSOUNAPI P, MP15-11, UP2-36

TSUCHIYA K, UP5-28

TSUCHIYA T, VS13-6

TSUDA M, MP33-13

TSUI J, MP20-15, MP22-6, UP3-24, UP3-25

TSUJII T, MP29-19

TSUKIMORI S, VS17-1

TSUKUDA F, UP3-1

TSUMURA H, MP15-20, MP20-6

TSUTSUMI S, UP5-35

TUCCI C, MP8-9, MP32-6

TUDERTI G, MP14-12, MP16-13, MP16-17, MP30-7, UP1-56, UP2-3, VS7-1, VS7-4, VS9-9, VS10-5, VS16-4, VS17-4, VS17-9

TUFEK I, VS7-7

TÜFEK I, MP6-11, MP16-2, MP18-1, MP20-10, UP1-48, VS9-7

TUGCU V, MP20-7, MP20-17, UP2-56, VS9-6

TUGRUL K, MP17-22

TUNA M, MP1-8, MP6-11, MP16-2, MP18-1, MP20-10, VS7-7, VS9-7

TUNACI A, MP4-4, MP4-5, UP1-36

TUNC L, MP3-8, MP20-9, MP33-16, VS3-7, VS3-8, VS10-7, VS13-8, VS13-10

TUNCAY O, MP32-13

TUNCEL A, MP17-7, MP24-4, MP29-20

TURAN T, MP25-8

TURHAN T, MP24-4

TURNEY BW, MP5-5, MP7-2, MP24-17, MP29-6, MP32-15

TURO-ANTONA J, MP16-20, MP25-3, UP5-3, VS14-13, VS17-5

TWISS CO, UP3-22

TYAGI S, VS8-7

TYAGI V, VS11-2, VS11-3, VS11-4, VS13-3, VS14-8

TZOU DT, MP7-7, MP10-18, MP28-3, MP33-2, MP33-3, UP1-53

$\mathbf{U}$

UCAR T, MP25-8

UCHIDA Y, MP29-19

UCHIO E, MP19-13

UDAONDO CASCANTE M, MP3-11

UEHARA S, MP9-18

UEMURA M, UP1-57

UETANI M, MP20-8
UJIKE T, UP1-57

UKIMURA O, MP16-16

ULKER V, UP6-9

UMEMOTO S, UP5-35

UMRANIKAR S, UP6-17

UNTERBERG S, VS8-9, VS12-5

UPPALURI N, MP11-16

URIBE C, MP12-22

URSINY M, MP28-22

USAWACHINTACHIT M, MP7-7

USLU M, MP26-10

UZAN A, MP2-5, UP1-21

UZZO R, MP16-13, MP23-6

\section{V}

VAGGERS SN, MP13-1

VALDEZ COLIN J, MP2-16

VALERIO M, MP6-1

VALLATI G, MP14-12, VS16-4, VS17-9

VALLEY ZA, MP5-21, UP1-19

VAN LAECKE E, MP9-7

VAN VELTHOVEN RF, VS5-2

VAN DE WIEL E, UP2-24

VANDE WALLE J, MP9-7

VARKARAKIS I, UP6-4

VARMI M, MP1-7

VASHISHT A, MP14-15

VASUDEVA P, VS11-2, VS11-3, VS11-4,

VS13-3, VS14-8

VAUGHAN LE, MP2-1, UP1-14

VEENSTRA B, VS18-11

VELA, I.T.C.A.S.T.I.L.L.O.A.C.U.T.E.N., VS14-13

VELTIEN A, UP2-24

VELUETA-MARTÍNEZ IA, UP6-14

VEMULAKANDA V, MP14-19

VENEZIANO D, MP6-8

VENKATESH R, MP8-2, MP8-3, MP21-7, MP25-7, MP31-12,

MP32-7, UP1-23, UP4-54, UP4-55

VENNERI BECCI A, MP11-5

VENZANO F, MP32-2, MP32-10

VEREP S, MP4-4, MP4-5, MP8-21, MP24-9, MP32-16, UP1-36, UP4-3

VERSOS R, VS11-1

VETTER J, MP4-7, MP4-8, MP8-2, MP8-3, MP16-12, MP18-11, MP21-7, MP23-12, MP25-7, MP30-19, MP31-12, MP32-7, UP1-23, UP4-54, UP4-55

VICENTINI F, MP26-20

VIERS BR, VS12-6, VS16-7, VS16-8

VIGUÉS F, MP7-11, UP4-46

VIJAY GANAPATHY SV, UP6-10

VIJAYAKUMAR M, MP7-6, MP18-2, VS12-1

VILA-REYES H, MP21-11, VS15-8

VILLANUEVA C, MP14-19

VINAROV A, MP30-21, VS4-7

VINCE RA, VS17-14

VINNICHENKO V, MP5-6, MP5-9

VIPRAKASIT D, MP10-12, MP28-3, MP28-20

VIRASORO R, MP3-12, MP22-12

VITALLI AS, VS8-3

VIZZIELLO D, MP11-22, MP27-17, UP3-16, UP3-28, UP3-58

VOGT B, UP6-47

VOLPI G, MP14-10, UP2-33, UP5-38

VOROBYEV A, VS9-12, VS17-10 
VOSS J, UP5-58, UP6-38

VRTISKA TJ, MP2-1, UP1-14

VU D, MP17-13, VS13-2

VURUSKAN H, VS14-10, VS15-6

W

WADA K, MP9-18, MP15-16, MP25-9, MP25-11, MP30-16, UP2-22, UP2-39, UP6-55

WAGENIUS M, MP29-18

WAGH Y, VS4-3

WAGNER H, MP7-3, MP7-4, MP15-1, UP2-31, UP4-1

WAGNER KR, MP24-10, MP33-15, UP3-29

WAGRELL L, MP6-15

WAHAFU W, MP23-8

WAIGANKAR SS, VS7-6, VS9-2, VS10-4, VS12-7, VS15-7, VS17-8, VS18-2

WAKUMOTO Y, MP28-11

WALDORF B, VS16-6, VS16-13

WALIA A, UP1-27

WALIA S, MP19-13, MP19-14

WALLACE S, UP3-6

WALMSLEY B, MP7-5

WAN F, MP23-1, UP5-4

WANG A, MP15-10, UP2-34

WANG G, MP17-8, MP20-18, UP2-29

WANG H, UP5-23

WANG J, MP11-6

WANG K, MP3-10, MP14-13, MP18-18, UP1-11, UP5-41, UP6-32

WANG L, UP1-29

WANG M, MP11-6, MP23-8

WANG S, MP1-12, MP9-22, MP26-21, MP29-17, MP33-22, UP4-27

WANG W, MP2-19, MP9-10

WANG X, MP1-10, MP9-22, MP17-10, UP2-58, UP4-28, UP5-36

WANG Y, MP5-1, MP19-7, MP20-14, MP22-10, MP31-2, MP33-11, UP3-37

WANG Z, MP1-12

WANG L, MP9-10

WANIS M, MP23-9

WANN CV, VS14-1

WARD JF, MP17-9

WARD R, MP4-11

WARNER A, UP5-20

WARNER RM, MP13-1

WASSERMAN M, MP8-9, MP32-6

WATANABE M, MP15-16, MP19-19, MP25-9, MP29-13, UP6-55

WATANABE R, MP9-18, UP2-43

WATANABE T, MP15-16, MP25-9, MP25-11, MP30-16, UP2-22, UP2-39

WATANUKI S, MP22-18

WEBB D, MP29-16

WEBER S, UP3-45

WEI T, UP5-18

WEI Y, MP1-21, UP2-42

WEIGAND K, UP5-17

WEIJERMAN P, UP2-57

WEINBERG A, MP14-8, VS15-4

WEINER D, MP31-17

WEISS B, MP17-14

WEISS H, MP5-3
WEN J, MP23-21

WEN S, UP3-9

WERNEBURG G, MP23-16, MP23-17

WETHERELL D, MP29-16

WHELAN P, MP22-16

WHITE C, VS6-13, VS7-5

WHITE E, UP5-13

WHITE W, MP23-6

WHITEHURST L, MP32-8, MP33-1

WHITING D, UP3-32

WIDICAHYONO S, MP26-13

WIENER C, MP10-12

WIENER S, MP10-18, MP33-2, MP33-3, UP1-53

WIESSMEYER JR, MP10-19, MP29-14

WIJBURG C, UP2-57

WILHELM K, MP22-9

WILKINSON M, MP7-3, MP15-1

WILLARD J, MP7-4, UP4-1

WILLIAMS M, MP11-17, MP13-7

WILLIAMS SC, MP8-16, UP1-54, VS12-3

WINER A, MP30-19

WINKLER H, MP10-22, UP4-51, UP5-31, UP6-59

WINOKER J, MP4-3, MP23-13, UP1-35

WINSHIP BB, MP10-20, MP27-18, MP28-1, MP29-12, UP1-55, UP6-1, VS10-8

WISEMAN O, MP7-2, MP7-12, MP7-22, MP8-11, MP24-17, MP24-21, MP32-15

WITHINGTON J, MP7-2, MP7-12, MP7-16, MP7-17, MP7-22, MP28-18, MP32-15, UP4-2, UP4-19, UP4-20

WOLF S, MP9-11

WOLLIN D, MP10-20, MP27-18, MP29-12, UP1-55, UP6-1, VS10-8

WONG J, MP30-14

WONG K, MP9-15, MP11-18, MP22-5

WONG P, MP9-17

WONG W, UP5-27

WOO H, MP11-17

WOOD K, MP2-20, MP2-21, MP10-21, UP6-40, UP6-57

WOODHAMS S, MP30-5, UP2-18

WORANISARAKUL V, MP8-14

WRIGHT C, VS6-8

WRIGHT T, MP8-9, MP32-6

WROCLAWSKI ML, UP1-1

WU C, MP20-14, MP28-3

WU H, UP5-18

WU J, VS14-12

WU R, UP2-25, VS9-3

WU T, VS7-13

WU W, MP8-22, MP12-10, MP13-4, MP26-8, MP33-5, UP6-5, UP6-36, UP6-51

WU Y, MP1-21, MP9-22, MP29-17, UP2-42

$\mathbf{X}$

XIA S, MP22-2

XIAO B, MP4-20, MP5-18, MP21-9, MP21-10, MP26-21, MP33-22, UP4-27

XIAO F, MP9-22

XIAO K, MP18-18, UP6-32

XING N, MP23-8, VS3-14, VS11-6, VS11-8, VS18-12

XU D, UP2-41, UP2-59, UP2-60

XU H, UP5-37

XU N, MP1-21, UP2-42

XU P, MP1-1 
XUE W, MP22-2

XUE X, MP1-21, UP2-42

XUN Y, MP9-22, MP29-17

Y

YADAV K, MP1-1

YADAV S, VS11-2, VS11-3, VS11-4, VS13-3, VS14-8

YAĞCI A, MP32-13

YAGHOUBIAN A, MP28-22

YAHIA M, VS14-5, VS14-9

YAKUPOGLU Y, UP5-46, VS6-2, VS8-4, VS13-1, VS13-7, VS14-11

YALCIN S, MP3-8, MP20-9, MP33-16, VS3-7, VS3-8, VS10-7, VS13-8, VS13-10

YALLAPPA S, MP31-9

YAMADA H, MP23-10

YAMADA Y, MP21-21, VS13-6

YAMAGUCHI K, MP33-13

YAMAMOTO H, MP22-3, MP25-22

YAMAMOTO Y, MP33-13

YAMAN O, MP6-2, MP33-18

YAMANE H, VS17-11

YAMASAKI M, UP2-49

YAMASAKI T, MP9-18

YAMAUCHI Y, MP23-10

YANAGIHARA Y, UP2-43

YANG B, MP18-21

YANG C, VS12-9

YANG DY, MP33-21, UP3-33, UP3-34, VS6-7, VS12-6, VS16-7, VS16-8

YANG F, MP23-8

YANG H, MP6-12

YANG K, VS16-6, VS16-10, VS16-13

YANG M, MP2-2

YANG W, MP1-17

YANG Y, VS7-14

YAO J, VS13-5

YAO M, MP16-1, MP19-10

YASSIN M, MP22-17

YASUI M, UP5-35

YAU J, MP17-14

YAVAN O, UP2-26

YAVASCAOGLU I, VS17-3

YAVUZ A, MP13-6

YAZAR VM, MP13-6

YE D, MP1-22, MP4-15

YE Z, MP22-10

YECIES T, MP4-19, MP22-17, MP33-6

YEE C, MP9-15, MP30-14

YEHIA M, MP21-5

YELLIN J, UP1-39

YENICE M, MP20-7, VS9-6

YEOW Y, MP19-22

YESILDAL C, MP32-9, UP3-18

YIGITBASI I, MP20-7, VS9-6

YILDIRIM A, MP25-8

YILMAZ A, UP5-46, VS6-2, VS13-7

YILMAZ S, VS10-7, VS13-8

YITGIN Y, MP4-4, MP4-5, UP1-36

YOKOYAMA N, MP20-20

YONEMORI M, MP17-11, MP21-21

YONG D, MP16-10, VS18-3

YONG J, VS4-5

YOO E, MP15-5, MP32-4
YOO J, MP10-4, MP26-3

YOO K, MP22-20

YOO S, MP13-15, MP29-15

YOON R, MP5-21, MP21-13, MP27-20

YOON S, MP6-13, UP3-53

YOON Y, MP3-16, MP33-7, UP4-13, VS2-10, VS5-13,

VS7-12, VS11-12, VS15-13

YOSHIDA K, MP19-19, MP19-20

YOSHIDA S, MP29-19

YOSHIDA T, MP14-20, VS12-12

YOSHINO H, MP17-11

YOSHIOKA T, MP9-18, MP15-18, UP2-19

YOSHIZUMI T, UP1-55

YOUNG A, MP18-8

YOUNG G, MP9-21

YOUNG M, MP19-9, MP28-15

YOUNIS N, UP5-5

YOUSEFI A, MP12-5

YU C, VS7-13

YU S, UP4-52

YUAN L, MP20-21

YUCETAS U, MP9-20

YUK H, MP13-3, UP1-7

YUMIOKA T, MP15-19, UP2-36, UP2-37, VS17-11

YUN J, MP1-17

YUTKIN V, UP1-46

YUWONO A, MP19-22

YUZHAKOV S, MP10-16

\section{$\mathbf{Z}$}

ZAFAR A, UP1-23

ZAIKIN A, MP24-13

ZAKOJI H, UP6-53

ZAKRI R, MP22-7

ZAMEL S, MP21-1

ZAMPINI A, MP2-12

ZANETTI G, MP14-11, VS15-1

ZANETTI S, MP5-7, MP22-1, MP31-3, UP1-44, UP1-45, UP4-8, VS15-3

ZARE A, MP32-1

ZARRELLI G, UP3-12, VS3-13, VS14-7

ZARZOUR M, UP5-17

ZATTONI F, MP5-2, MP21-4

ZEECK K, VS9-4

ZELL M, UP3-11

ZENG G, MP7-10, MP8-22, MP13-4, MP19-2, MP19-15,

UP1-25, UP1-33, UP4-6

ZENNAMI K, MP29-13

ZETUMER S, UP1-53

ZEYNALOVA G, UP3-18

ZHANG B, UP5-23

ZHANG C, MP17-8, MP20-18, UP2-29

ZHANG G, MP1-20, MP20-19, UP1-32, UP2-44

ZHANG L, UP6-25, VS14-12

ZHANG W, MP15-12

ZHANG X, MP4-20, MP5-18, MP21-9, MP21-10, MP26-21,

MP33-22, UP1-26, UP4-27

ZHAO LC, MP14-8, MP14-9, VS15-4, VS16-5

ZHAO Z, MP13-4

ZHENG Q, MP1-21, UP2-42

ZHONG H, MP22-2

ZHONG L, UP2-17

ZHONG S, UP6-7

ZHONG W, MP13-4 
ZHOU A, MP22-13

ZHOU X, MP17-8, MP20-18, UP2-29

ZHU G, UP3-35

ZHU Q, MP20-21

ZHU W, MP13-4, UP1-25, UP1-33

ZHU Y, MP1-22, MP4-15, MP29-17

ZHUO J, MP11-8

ZIEMBA JB, MP13-5, UP3-48
ZILBERMAN DE, MP10-22, UP4-51, UP5-31, UP6-59

ZIOGAS A, UP3-50

ZIOUIOU I, VS2-7, VS8-11

ZIPUNNIKOV V, VS13-9, VS14-4

ZISMAN A, UP1-31, UP2-5

ZOR M, UP2-26

ZORN KC, UP3-23

ZULUAGA L, MP3-7, UP1-6 